\title{
Numerical and Experimental Comparison Among a New Hybrid FT-Music Technique and Existing Algorithms for Through-the-Wall Radar Imaging
}

\author{
Renato Cicchetti, Senior Member, IEEE, Stefano Pisa, Senior Member, IEEE, Emanuele Piuzzi, \\ Member, IEEE, Erika Pittella, Member, IEEE, Paolo D'Atanasio, and Orlandino Testa
}

\begin{abstract}
A fast low-cost through-the-wall radar imaging (TWRI) system, based on a vector network analyzer (VNA), a couple of switches and an array of Vivaldi antennas, has been designed, realized, and tested. To solve the TWRI inversion problem, an original theoretical modeling for a class of TWRI techniques whose basic functions are the cross-range Fourier Transform of the scattered field and its covariance operator has been proposed. Using these functions, four conventional algorithms, namely the delay and sum (DAS), the Fourier Transform (FT), the Multiple Signal Classification (MUSIC), the hybrid DAS-MUSIC and a new algorithm, the hybrid FT-MUSIC, have been derived. All these techniques have been implemented and their accuracy and field of view have been tested on canonical scatterers. Then, the algorithms have been applied to measured data collected in different scenarios constituted by a metallic bar or a human subject in absence and in presence of a wall between the antenna and the considered targets. Using the proposed TWRI system, it has been possible to detect a subject located up to 5-m away from the Radar antenna array through a tuff wall. The proposed FT-MUSIC algorithm has evidenced performances similar to those of the DAS-MUSIC but with significantly lower execution times. Finally, FT-MUSIC performances in terms of field of view and immunity to disturbances are better compared to those of the MUSIC algorithm.
\end{abstract}

Index Terms-Delay and sum (DAS), delay and sum - multiple signal classification (DAS-MUSIC), Fermat's principle, Fourier transform (FT), Fourier transform - multiple signal classification (FT-MUSIC), multiple signal classification (MUSIC), throughthe-wall radar imaging (TWRI) system, Vivaldi antennas.

\section{INTRODUCTION}

$\mathrm{T}$ HROUGH-THE-WALL radar imaging (TWRI) systems are used by police, fire personnel and defense forces to detect, identify and track subjects inside buildings or under rubble [1], [2]. TWRI systems are generally based on frequency modulated continuous wave (FMCW) [3]-[6], ultra-wideband (UWB) [7]-[10] or stepped-frequency (SF) radars [11]-[17]. All these radars send an RF field towards the target and acquire the reflected signal. Then suitable algorithms are applied on the received signals in order to reconstruct an image of the

This work was supported in part by the Italian Ministry of University and Research under the PRIN 2015: U-VIEW (Ultra-wideband Virtual Imaging Extra Wall for high-penetration high quality imagery of enclosed structures).

R. Cicchetti, S. Pisa, E. Piuzzi, and O. Testa are with the Department of Information Engineering, Electronics and Telecommunication of Sapienza University of Rome, 00184 Rome, Italy (e-mail: renato.cicchetti@)uniroma1.it; investigated scenario. In particular, the range migration (RM) algorithm applied to FMCW or SF radar data was used in [3], [4] and [16], while the back projection time domain algorithm was implemented on UWB data in [9], on FMCW radar data in [6], and on SF data in [16]. The compressive sensing technique applied to the signals received by a stepped-frequency radar was proposed in [14], [15]. Finally, the super resolution MUSIC algorithm was used in [11] and [17]. All these papers dealt with the theoretical basis of a specific reconstruction technique, while little effort has been done to understand the common background of the various techniques and to perform comparisons so as to evaluate pro and cons of each technique with respect to the others.

Concerning the experimental setups, these generally consist of an RF source, an amplifier, a receiver stage and transmitting (Tx) and receiving (Rx) antennas. In some labs Tx and Rx horn antennas were mounted on a field probe scanner [11], [12], on a 1D rail [3], or were manually moved at fixed positions [6], [16]. A more effective experimental set-up was developed at Lincoln-MIT laboratory [4]. This system employs 21 Vivaldi antennas, controlled by 4 solid state and digitally controlled switches. The antennas are mounted on a box structure at fixed positions. The transmitting channel utilizes 13 antennas while eight antennas are used in the receiving one obtaining a 44 element virtual array evenly spaced at $\lambda / 2$. This system is based on a FMCW radar architecture with a center frequency of 3 $\mathrm{GHz}$ and a $2 \mathrm{GHz}$ chirp. The peak transmitted power is $1 \mathrm{~W}$ with a $50 \%$ duty cycle. At the Army Research Laboratory (ARL) a UWB radar based on two transmitting TEM horns antennas and 16 receiving Vivaldi antennas was realized [7], [8]. This system, which operates between 0.3 and $3 \mathrm{GHz}$ with an average power across the bandwidth of $5 \mathrm{~mW}$, was assembled on a supporting structure mounted on the roof of a van. Another UWB system, realized at the University of Tennesse [9], covers the 2-6 GHz band with one transmitting and eight receiving Vivaldi antennas connected to a single pole eight throw (SP8T) RF switch. The system is able to transmit a power of $25 \mathrm{dBm}$ with a receiver sensitivity of $-65 \mathrm{dBm}$. The

stefano.pisa@uniroma1.it; orlandino.testa@uniroma1.it).

emanuele.piuzzi@uniroma1.it;

P. D'Atanasio, is with Italian National Agency for New Technologies, Energy and Sustainable Economic Development, Casaccia Research Centre, Rome 00123, Italy (e-mail: paolo.datanasio@enea.it).

E. Pittella is with Department of Legal and Economic Sciences, Pegaso University, 00186, Rome, Italy (e-mail: erika.pittella@unipegaso.it). 
above mentioned systems, very often, utilize commercial and expensive antennas like horn or TEM horn together with dedicated and expensive RF sources and receivers.

In this paper, a theoretical model for a class of TWRI reconstruction algorithms is presented. Then a low-cost and fast radar system for TWRI is proposed. The radar, based on SF topology, employs commercial switches (SP4T) and a set of Vivaldi antennas. The system is controlled by a LabVIEW program suitable of driving switches and acquiring data from a vector network analyzer (VNA). This instrument is almost always available in a microwave lab. Alternatively, since the maximum frequency for this application is lower than $4 \mathrm{GHz}$, it is possible to employ a fairly inexpensive instrument based on USB VNA technology. Finally, delay and sum (DAS), Fourier Transform (FT), Multiple Signal Classification (MUSIC), DAS-MUSIC and the new FT-MUSIC algorithms will be implemented and applied in a scenario composed of scatterers located in free space or behind a brick wall. A preliminary feasibility study is performed in order to establish the best frequency range and the required RF output power for the best scenario reconstruction.

\section{FEAsibility StUdy}

\section{A. System Power Budget}

Fig. 1 shows the schematic of a SF radar placed in front of a building with a human subject (scatterer) located inside. The vector network analyzer (VNA) measures, in a given number of equally spaced frequency points, the scattering parameters between a transmitting (Tx) and a receiving ( $\mathrm{Rx}$ ) antenna selected by the two switch systems. In this manner, an equivalent antenna whose phase center is located between $\mathrm{Tx}$ and Rx is achieved [4]. A power amplifier (PA) can be inserted in the transmitting channel to increase the Radar range.

Fig. 2 shows a simplified model of the investigated scenario, which takes into account the equivalent antenna, the wall of thickness "d", and the scatterer placed at a distance R from the antenna. For the system power budget at the working frequency $\mathrm{f}$, the radar equation can be used:

$$
P_{R}=P_{T} \sigma(f)|T(f)|^{4} \frac{G(f)^{2}}{4 \pi}\left[\frac{\lambda}{(4 \pi R)^{2}}\right]^{2} .
$$

This equation relates the received power $P_{R}$ to the transmitted one $P_{T}$, the antenna gain $G(f)$, the free-space wavelength $\lambda$, the target distance $R$, the scatterer radar cross section (RCS) $\sigma(f)$, and the wall transmission $T(f)$ coefficient.

In particular, the $T$ coefficient can be expressed as follows:

$$
T=\frac{2}{A+\frac{B}{\eta_{0}}+\eta_{0} C+D}
$$

where $\eta_{0}$ is the free space wave impedance while $\mathrm{A}, \mathrm{B}, \mathrm{C}$ and $\mathrm{D}$ are the transmission matrix coefficients given by:

$$
\left[\begin{array}{ll}
A & B \\
C & D
\end{array}\right]=\left[\begin{array}{cc}
\cosh \left(\gamma_{m} d\right) & Z_{m} \sinh \left(\gamma_{m} d\right) \\
\frac{\sinh \left(\gamma_{m} d\right)}{Z_{m}} & \cosh \left(\gamma_{m} d\right)
\end{array}\right]
$$

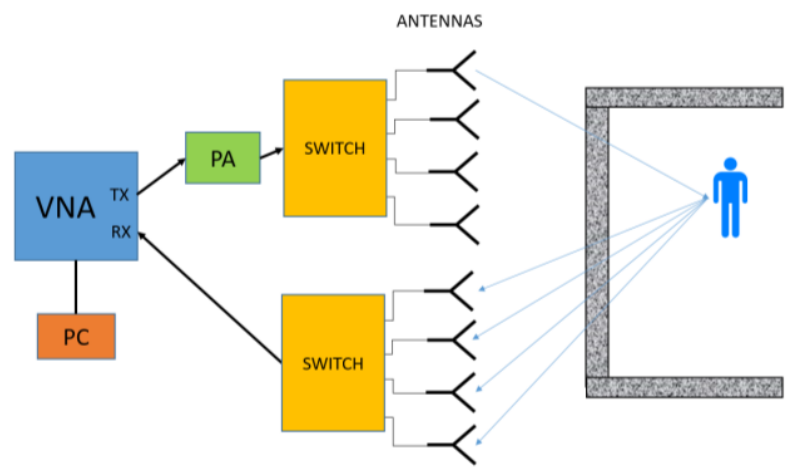

Fig. 1. Schematic of a SF radar placed in front of a building with a human subject (scatterer) located inside.

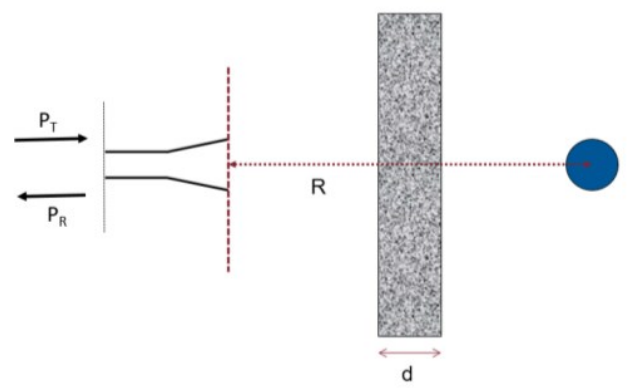

Fig. 2. Simplified model of the investigated scenario.

with

$$
\gamma_{m}=j \frac{\omega}{c} \sqrt{\varepsilon_{r}^{\prime}-j \varepsilon_{r}^{\prime \prime}}
$$

where $\omega$ is the angular frequency, $Z_{m}$ and $\gamma_{m}$ are the wall material wave impedance and propagation constant, $\mathrm{c}$ is the free space speed of light, while $\varepsilon_{r}^{\prime}$ and $\varepsilon_{r}^{\prime \prime}$ are the real and imaginary part of the wall complex relative permittivity.

\section{B. Feasibility Study Results}

The power budget for an operative scenario consisting of a wall with a thickness of $25 \mathrm{~cm}$ and a human target located $5 \mathrm{~m}$ far from the antenna has been evaluated using (1). The wall relative permittivity at around $5 \mathrm{GHz}$ ranges from 3.5 -j0.03 for dried wall to 7.5 -j0.5 for walls with a $5 \%$ gravimetric moisture content [18], [19]. To extrapolate the permittivity frequency behavior between 1 and $10 \mathrm{GHz}$, the data in [18] have been fitted using the Debye equation. The human body RCS is available both from simulations [20] and measurements [21]. Here the experimental results from [21] have been used. Concerning the antenna gain a value of $7 \mathrm{dBi}$ has been assumed.

Fig. 3 shows the received power as a function of frequency for a transmitted power level of $0 \mathrm{dBm}$. The received power reduces as the frequency increases and in the presence of moisture exhibiting strong attenuations for frequencies above 3 $\mathrm{GHz}$ where a wall with $5 \%$ moisture content gives rise to received powers lower than $-130 \mathrm{dBm}$. Moreover, the figure evidences received power frequency oscillations due to the frequency behavior of the human body RCS. 


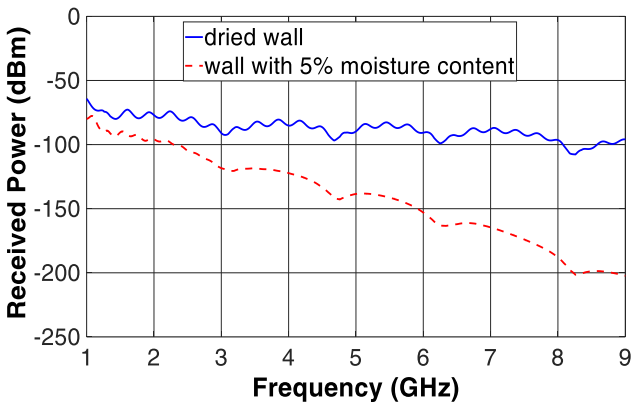

Fig. 3. Radar received power for the scenario depicted in Fig. 2. The transmitted power is equal to $0 \mathrm{dBm}$.

In conclusion, the 1-3 GHz frequency band seems to be the most suitable for TWRI applications. In particular, using a VNA with a dynamic range of $100 \mathrm{~dB}$ and a power amplifier of $20 \mathrm{~dB}$, frequencies up to $3 \mathrm{GHz}$ can be used for through the wall identification of human targets located up to $5 \mathrm{~m}$ far from the radar antennas. Obviously, greater distances can be covered by increasing the gain of the amplifier chain.

\section{THEORY FOR A CLASS OF TWRI MODELS}

The 2D TWRI problem described in the previous section is solved by means of a class of inversion models based on the cross-range spatial Fourier Transform of the received scattered field. As reported in the 2D scenario depicted in Fig. 4, $N_{T}$ point-like targets, located at $\left(x_{t_{q}}, y_{t_{q}}\right)$ and belonging to the region of interest (ROI) having center coordinates $\left(x_{0}, y_{0}\right)$, are illuminated by a field excited by an infinite array of $z$-oriented line sources located along $y=y_{p}$. Each target is characterized by its scattering term $\sigma_{q}(\mathrm{~S} \cdot \mathrm{m})$. Without loss of generality, the conditions $y_{0}>y_{\mathrm{p}}$ and $y_{t_{q}}>y_{\mathrm{p}}$ will be considered.

To expose the targets to a quasi-plane wave, the infinite $z$ oriented line source array reported in Fig. 4 is excited by means of the following linear electric current density distribution:

$$
\begin{aligned}
J_{e_{A}}(\omega, x, y)= & J_{e_{0}} \frac{y_{0}-y_{p}}{\sqrt{\left(x_{p}-x_{0}\right)^{2}+\left(y_{p}-y_{0}\right)^{2}}} \times \\
& \times e^{-j \frac{\omega}{c} \frac{\left(x_{p}-x_{0}\right)\left(x_{p}-x\right)}{\sqrt{\left(x_{p}-x_{0}\right)^{2}+\left(y_{p}-y_{0}\right)^{2}}}} \delta\left(y-y_{p}\right)
\end{aligned}
$$

where $J_{e_{0}}$ is the amplitude coefficient, $\delta(\cdot)$ is the Dirac pulse, while $\left(x_{p}, y_{p}\right)$ is the location of the phase center of the array where the scattered signal is received. The z-component of the incident electric field $E_{z}$ that impinges on the $q$-th target can be approximated by the following expression obtained by the convolution of the 2D free-space Green's function [22] with the current distribution (5):

$$
E_{z_{p}}^{i n}\left(\omega, x_{t_{q}}, y_{t_{q}}\right) \cong-\frac{1}{2} \eta_{0} J_{e_{0}} e^{-j \frac{\omega}{c} \sqrt{\left(x_{p}-x_{t_{q}}\right)^{2}+\left(y_{p}-y_{t_{q}}\right)^{2}}}
$$

Using (6), the $q$-th target scattered field received at $\left(x_{p}, y_{p}\right)$ can be express, under the assumption that $\sigma_{q}$ is angular

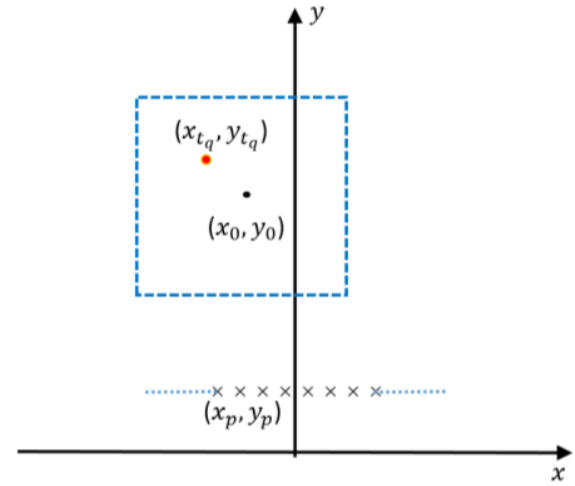

Fig. 4. Geometry of the considered scenario where a point-like target located at $\left(x_{t_{q}}, y_{t_{q}}\right)$ is reported. The $\left(x_{0}, y_{0}\right)$ point indicates the center of the region of interest (ROI) outlined by a dashed blue line. In the figure, the generic antenna of the array system, located at $\left(x_{p}, y_{p}\right)$, is also shown.

independent, as follows:

$$
\begin{aligned}
& E_{z_{q}}\left(\omega, x_{p}\right)=\left[-\frac{1}{2} \eta_{0} J_{e_{0}} e^{-j \frac{\omega}{c} \sqrt{\left(x_{p}-x_{t_{q}}\right)^{2}+\left(y_{p}-y_{t_{q}}\right)^{2}}}\right] \times \\
& \times \sigma_{q}\left[-\frac{|\omega|}{4 c} \eta_{0} H_{0}^{(u)}\left(\frac{|\omega|}{c} \sqrt{\left(x_{p}-x_{t_{q}}\right)^{2}+\left(y_{p}-y_{t_{q}}\right)^{2}}\right)\right]
\end{aligned}
$$

where $H_{0}^{(u)}$ is the Hankel function of the first or second kind $(u=1,2)$ and order zero. In (7) $u=1$ or $u=2$ if $\omega<0$ or $\omega>0$, respectively. Note that, the absolute operator $|\cdot|$ is introduced in (7) to account for the negative values of the angular frequency as required by the subsequent analytical developments.

To derive a class of TWRI models suitable to detect the location of scattering objects, it is useful to use the following approximate expression of (7), whose accuracy improves as the ROI center moves away from the array:

$$
\begin{aligned}
& E_{z_{q}}\left(\omega, x_{p}\right) \cong \\
& \cong \sqrt{2} \frac{\sigma_{q} \eta_{0}^{2}|\omega| J_{e_{0}}}{8 c} H_{0}^{(u)}\left(2 \frac{|\omega|}{c} \sqrt{\left(x_{p}-x_{t_{q}}\right)^{2}+\left(y_{p}-y_{t_{q}}\right)^{2}}\right)
\end{aligned}
$$

where the term $\sqrt{2}$ derives from doubling the argument of the Hankel function. Therefore, the total scattered field at the generic array receiver located at $\left(x_{p}, y_{p}\right)$ is given by:

$$
\begin{aligned}
& E_{z_{S}}\left(\omega, x_{p}\right)=\sum_{q=1}^{N_{T}} E_{z_{q}}\left(\omega, x_{p}\right) \cong \\
& \cong \sqrt{2} \frac{\eta_{0}^{2}|\omega| J_{e_{0}}}{8 c} \sum_{q=1}^{N_{T}} \sigma_{q} H_{0}^{(u)}\left(2 \frac{|\omega|}{c} \sqrt{\left(x_{p}-x_{t_{q}}\right)^{2}+\left(y_{p}-y_{t_{q}}\right)^{2}}\right)
\end{aligned}
$$

where $N_{T}$ is the number of targets illuminated by the RF field.

In many practical situations, when an array with a few number of elements is used, the excitation of the single array element located at $\left(x_{p}, y_{p}\right)$ can be used instead of the profile excitation 
(5). In any case, an approximation similar to that reported in (8) can be effectively adopted to model the scattered field in the receiving point $\left(x_{p}, y_{p}\right)$. In this case (9) will provide a less precise estimate of the field near the array region.

The key function of the proposed models is the cross-range Fourier Transform of the received scattered field, centered with respect to the ROI abscissa $x_{0}$, given by the following expression:

$$
\tilde{L}_{z_{S}}(\cdots,+x)=\int_{-\infty}^{+\infty} E_{z_{S}}(\omega, \xi) e^{-j \psi_{x}\left(\xi-x_{0}\right)} d \xi .
$$

Starting from (10) and using (9), a closed form of $\tilde{L}_{z_{S}}$ can be obtained as follows

$$
\begin{aligned}
& \left.\tilde{\Sigma}_{z_{S}, \ldots, x}\right)=\sqrt{2} \frac{\eta_{0}^{2}|\omega| J_{e_{0}}}{4 c} \frac{1}{\sqrt{\left(2 \frac{\omega}{c}\right)^{2}-\psi_{x}^{2}}} \times
\end{aligned}
$$

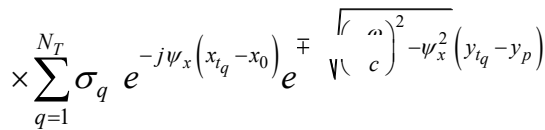

where the upper sign takes when $2 \frac{\omega}{c}>\left|\psi_{x}\right|$ and the lower sign when $2 \frac{\omega}{c}<-\left|\psi_{x}\right|$. Note that the branch point in (11) refers to the presence of an electric field wavenumber [see Eq. (9)] whose value $2 \frac{\omega}{c}$ is due to the round-trip propagation process of the electric field emitted by the array in the presence of scatterers.

A first model, referred to as FT based model, can be derived operating an analytical integration over the angular frequency $\omega$ useful to perform the integration of the non-evanescent spectral scattered electric field contribution with respect to the transverse wavenumbers $\psi_{x}$ and $\psi_{y}$, obtaining:

$$
\begin{aligned}
& I_{F T}(x, y)= \\
& \left.=\frac{1}{2 c} \int_{-\infty}^{\infty} \int_{\left|\psi_{x}\right|}^{\infty} \tilde{L}_{z_{S}}, \ldots, r_{x}\right) e^{j \psi_{x}\left(x-x_{0}\right)} e^{j \sqrt{\left(2 \frac{\omega}{c}\right)^{2}-\psi_{x}^{2}}\left(y-y_{p}\right)} d \omega d \psi_{x}+ \\
& +\int_{-\infty}^{\infty} \int_{-\infty}^{-\left|\psi_{x}\right|} \tilde{L}_{z_{S}\left(\ldots, r_{x}\right)} e^{j \psi_{x}\left(x-x_{0}\right)} e^{-j \sqrt{\left(2 \frac{\omega}{c}\right)^{2}-\psi_{x}^{2}\left(y-y_{p}\right)}} d \omega d \psi_{x}
\end{aligned}
$$

then, introducing the following new variables based on the separability condition:

$$
\left\{\begin{array}{l}
\psi_{x}^{\prime}=\psi_{x} \\
\psi_{y}^{\prime}=\operatorname{sgn}(\omega) \sqrt{\left(2 \frac{\omega}{c}\right)^{2}-\psi_{x}^{2}}
\end{array}\right.
$$

the FT intensity function (12) assumes the form of the inverse Fourier Transform:

$$
\begin{aligned}
& I_{F T}(x, y)= \\
& =\frac{1}{(2 \pi)^{2}} \int_{-\infty}^{\infty} \int_{-\infty}^{\infty} \tilde{L}_{z_{S}}\left(2^{-\sigma^{--}\left(\psi_{y}^{\prime}\right)} \sqrt{\psi_{x}^{\prime 2}+\psi_{y}^{\prime 2}}, \psi_{x}^{\prime}\right) e^{j \psi_{y}^{\prime}\left(y_{0}-y_{p}\right)} \times \\
& \quad \times e^{j \psi_{x}^{\prime}\left(x-x_{0}\right)} e^{j \psi_{y}^{\prime}\left(y-y_{0}\right)} W_{F T}\left(\psi_{x}^{\prime}, \psi_{y}^{\prime}\right) d \psi_{x}^{\prime} d \psi_{y}^{\prime}
\end{aligned}
$$

where

$$
W_{F T}\left(\psi_{x}, \psi_{y}\right)=\frac{\pi^{2}\left|\psi_{y}\right|}{\sqrt{\psi_{x}^{2}+\psi_{y}^{2}}}
$$

and $\operatorname{sgn}(\cdot)$ is the sign operator. The integrand (14) has been multiplied by $e^{-j \psi_{y}^{\prime} y_{0}}$ and $e^{+j \psi_{y}^{\prime} y_{0}}$ so as to express the inverse Fourier transformation with respect to the ROI center. Using (11), the integral in (14) is obtained as:

$$
I_{F T}(x, y)=\sqrt{2} \frac{\eta_{0}^{2} J_{e_{0}}}{8} \pi^{2} \sum_{q=1}^{N_{T}} \sigma_{q} \delta\left(x-x_{t_{q}}\right) \delta\left(y-y_{t_{q}}\right)
$$

From (16), it appears that the intensity function $I_{F T}(x, y)$ is suitable to identify the targets locations.

Using the large-argument asymptotic representation of the Hankel functions and the following analytical approximation of the electric distance between the observation point located in $(x, y)$ and the receiver located in $\left(x^{\prime}, y_{p}\right)$ of the antenna array

$2 \frac{\omega}{c} \sqrt{\left(x^{\prime}-x\right)^{2}+\left(y_{p}-y\right)^{2}} \cong$

$\cong 2 \frac{\omega}{c} \sqrt{\left(x^{\prime}-x_{o}\right)^{2}+\left(y_{p}-y_{o}\right)^{2}}+\xi_{x}\left(x-x_{o}\right)+\xi_{y}\left(y-y_{o}\right)$

where:

$$
\left\{\begin{array}{l}
\xi_{x}=-2 \frac{\omega}{c} \frac{x^{\prime}-x_{0}}{\sqrt{\left(x^{\prime}-x_{0}\right)^{2}+\left(y_{p}-y_{0}\right)^{2}}} \\
\xi_{y}=-2 \frac{\omega}{c} \frac{y_{p}-y_{0}}{\sqrt{\left(x^{\prime}-x_{0}\right)^{2}+\left(y_{p}-y_{0}\right)^{2}}}
\end{array}\right.
$$

the cross-range spectral field $\widetilde{E}_{z_{S}}(11)$ can be approximated as follows:

$\tilde{L}_{z_{S}}, \cdots, \supset_{x}, \cong$

$$
\begin{aligned}
& \cong E_{z_{S}}\left(\omega, x^{\prime}\right) e^{j 2 \frac{\omega}{c} \frac{\left(x^{\prime}-x_{0}\right)^{2}}{\sqrt{\left(x^{\prime}-x_{0}\right)^{2}+\left(y_{p}-y_{0}\right)^{2}}}} \times \\
& \times \sqrt{\frac{\pi c}{j \omega}} \frac{\left[\left(x^{\prime}-x_{0}\right)^{2}+\left(y_{p}-y_{0}\right)^{2}\right]^{3 / 4}}{\left|y_{0}-y_{p}\right|}
\end{aligned}
$$


It can be noted that the accuracy of (17) and (19) improves as the ROI center moves away from the array.

Replacing (19) in (14), and using (17)-(18), an approximate DAS intensity function, which is based on the array received field (9), is derived:

$$
\begin{aligned}
I_{D A S}(x, y) & =\frac{1}{c} \int_{-\infty}^{\infty} \int_{-\infty}^{\infty} E_{z_{S}}\left(\omega, x^{\prime}\right) e^{j 2 \frac{\omega}{c} \sqrt{\left(x^{\prime}-x\right)^{2}+\left(y_{p}-y\right)^{2}}} \times \\
& \times W_{D A S}\left(\omega, x^{\prime}\right) d \omega d x^{\prime}
\end{aligned}
$$

where:

$$
W_{D A S}\left(\omega, x^{\prime}\right)=\sqrt{\frac{\pi \omega}{j c}} \frac{\left|y_{0}-y_{p}\right|}{\left[\left(x^{\prime}-x_{0}\right)^{2}+\left(y_{p}-y_{0}\right)^{2}\right]^{\frac{3}{4}}}
$$

is the corresponding complex weight function.

Eq. (14) can be also used to derive TWRI MUSIC type algorithms based on the spectral eigenvectors decomposition. To this end, the covariance operator $L_{\psi_{x}, \psi_{y}}^{\psi_{x}^{\prime}, \psi_{y}^{\prime}}$ based on the crossrange Fourier Transform of the received scattered field, is defined as in (22) at the bottom of the page. After some algebraic manipulation, it can be verified that the operator (22) has eigenvalues whose expression can be identified by means of (23) at the bottom of the page where

$$
\tau_{\psi_{x}, \psi_{y}}(x, y)=e^{-j \psi_{x}\left(x-x_{0}\right)} e^{-j \psi_{y}\left(y-y_{0}\right)}
$$

and

$$
\Phi(\xi)= \begin{cases}1 & \xi=0 \\ 0 & \xi \neq 0\end{cases}
$$

Equation (23) reveals the Hermitian nature of the operator $L_{\psi_{x}, \psi_{y}}^{\psi_{x}^{\prime}, \psi_{y}^{\prime}}$ having complex eigenfunctions $\tau_{\psi_{x}, \psi_{y}}(x, y)$ and eigenvalues $\lambda_{q}$ as not negative real quantities. In particular, the non-vanishing eigenvalues take the following expression when $x=x_{t_{q}}$ and $y=y_{t_{q}}$

$\lambda_{q}=\frac{\pi^{4} \eta_{0}^{4}\left|J_{e_{0}}\right|^{2}}{32}\left|\sigma_{q}\right|^{2} \quad$ with $q=1, \ldots, N_{T}$

Then, introducing the auxiliary operator $M$ which projects a generic function in the space spanned by the null-eigenvalue eigenfunctions $\tau_{\psi_{x}, \psi_{y}}(x, y)$,

$$
M_{\psi_{x}, \psi_{y}}^{\psi_{x}^{\prime}, \psi_{y}^{\prime}}=\delta_{\psi_{x}, \psi_{y}}^{\psi_{x}^{\prime}, \psi_{y}^{\prime}}-\sum_{q=1}^{N_{T}} \tau_{\psi_{x}, \psi_{y}}\left(x_{t_{q}}, y_{t_{q}}\right) \tau_{\psi_{x}^{\prime}, \psi_{y}^{\prime}}^{*}\left(x_{t_{q}}, y_{t_{q}}\right)
$$

where $\delta_{\psi_{x}, \psi_{y}}^{\psi_{x}^{\prime}, \psi_{y}^{\prime}}$ is the identity operator defined as

$$
\delta_{\psi_{x}, \psi_{y}^{\prime}, \psi_{x, r_{y}}^{\prime}}^{\psi_{y}^{\prime}} \quad g_{\psi_{x}, \psi_{y}}
$$

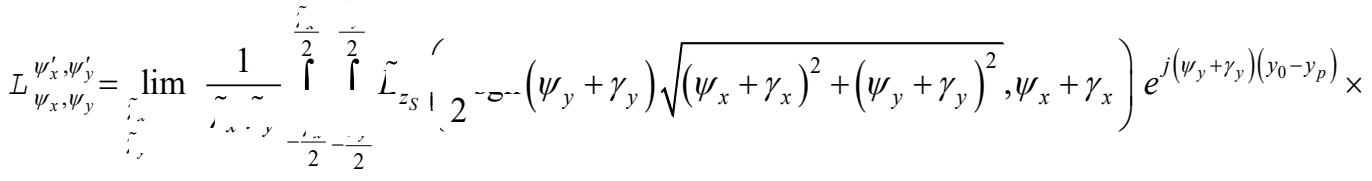

$$
\begin{aligned}
& \times \tilde{\Sigma}_{z_{S}}\left(2^{-\sigma^{--}}\left(\psi_{y}^{\prime}+\gamma_{y}\right) \sqrt{\left(\psi_{x}^{\prime}+\gamma_{x}\right)^{2}+\left(\psi_{y}^{\prime}+\gamma_{y}\right)^{2}}, \psi_{x}^{\prime}+\gamma_{x}\right) e^{-j\left(\psi_{y}^{\prime}+\gamma_{y}\right)\left(y_{0}-y_{p}\right)} \times \\
& \times W_{F T}\left(\psi_{x}+\gamma_{x}, \psi_{y}+\gamma_{y}\right) W_{F T}\left(\psi_{x}^{\prime}+\gamma_{x}, \psi_{y}^{\prime}+\gamma_{y}\right) d \gamma_{x} d \gamma_{y}
\end{aligned}
$$

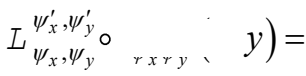

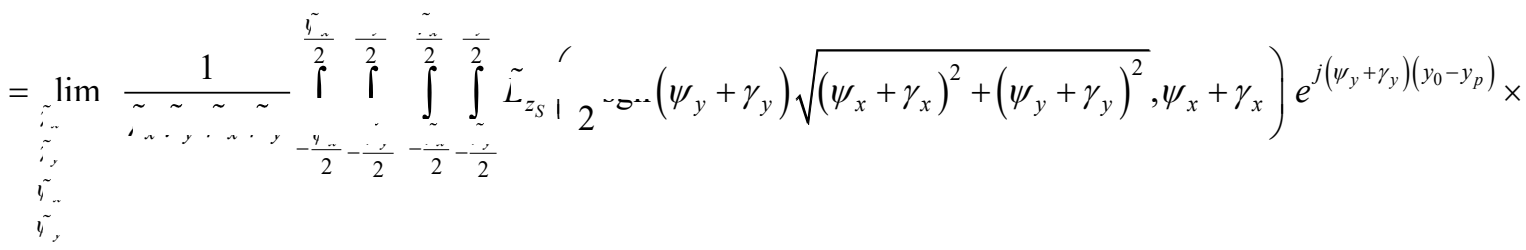

$$
\begin{aligned}
& \times \tilde{\bar{L}}_{z_{S}}\left(2^{-\sigma^{--}}\left(\psi_{y}^{\prime}+\gamma_{y}\right) \sqrt{\left(\psi_{x}^{\prime}+\gamma_{x}\right)^{2}+\left(\psi_{y}^{\prime}+\gamma_{y}\right)^{2}}, \psi_{x}^{\prime}+\gamma_{x}\right) e^{-j\left(\psi_{y}^{\prime}+\gamma_{y}\right)\left(y_{0}-y_{p}\right)} \times \\
& \times W_{F T}\left(\psi_{x}+\gamma_{x}, \psi_{y}+\gamma_{y}\right) W_{F T}\left(\psi_{x}^{\prime}+\gamma_{x}, \psi_{y}^{\prime}+\gamma_{y}\right) e^{-j \psi_{x}^{\prime}\left(x-x_{0}\right)} e^{-j \psi_{y}^{\prime}\left(y-y_{0}\right)} d \gamma_{x} d \gamma_{y} d \psi_{x}^{\prime} d \psi_{y}^{\prime}= \\
& =\frac{\pi^{4} \eta_{0}^{4}\left|J_{e_{0}}\right|^{2}}{32} \sum_{q=1}^{N_{T}}\left|\sigma_{q}\right|^{2} \tau_{\psi_{x}, \psi_{y}}(x, y) \Phi\left(x-x_{t_{q}}\right) \Phi\left(y-y_{t_{q}}\right)
\end{aligned}
$$


while the dot operator is defined by:

$$
\begin{aligned}
& f_{\psi_{x} \psi_{y}}{ }^{\circ}, \ldots,
\end{aligned}
$$

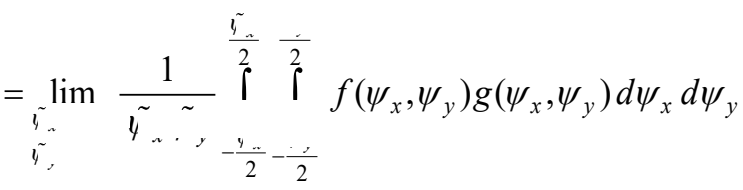

the FT MUSIC intensity function $I_{\text {FTMUSIC }}(x, y)$ is defined as follows [11]:

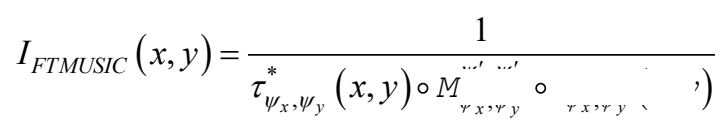

The $I_{\text {FTMUSIC }}(\cdot)$ function allows the identification of the targets position as it exhibits a peak for each of the $N_{T}$ eigenfunctions having non-zero eigenvalue due to the vanishing of its denominator. Note that according (29), eigenfunctions $\tau_{\psi_{x}, \psi_{y}}(x, y)$ have unitary norm, i.e $\left.\tau_{\psi_{x}, \psi_{y}}(x, y) \circ_{\tau_{x}, \tau_{y},}^{*}, y\right)=1$.

Different MUSIC based intensity functions can be derived choosing covariance operators $L_{\psi_{x}, \psi_{y}}^{\psi_{x}^{\prime}, \psi_{y}^{\prime}}$ different from that defined in (22). For instance, using (19), an approximated operator $L_{\psi_{x}, \psi_{y}}^{\psi_{x}^{\prime}, \psi_{y}^{\prime}}$ is obtained, referred as basic MUSIC covariance operator [11], reported in (31) at the bottom of the page with

$$
E_{z_{S}}^{c}(\omega, x)=E_{z_{S}}(\omega, x) e^{j 2 \frac{\omega}{c} \sqrt{\left(x^{\prime}-x_{0}\right)^{2}+\left(y_{p}-y_{o}\right)^{2}}}
$$

and

$$
W^{\prime}\left(\psi_{y}\right)=\pi^{2} \sqrt{\frac{2 \pi\left|y_{0}-y_{p}\right|}{j \psi_{y}}}
$$

Finally, observing that the 2D Fourier transform of (14) gives

$$
\begin{aligned}
& \int_{-\infty}^{\infty} \int_{-\infty}^{\infty} I_{F T}\left(x^{\prime}, y^{\prime}\right) e^{-j \psi_{x}\left(x^{\prime}-x_{0}\right)} e^{-j \psi_{y}\left(y^{\prime}-y_{0}\right)} d x^{\prime} d y^{\prime}= \\
& \tilde{L}_{z_{S}}\left(2^{-\infty-\left(\psi_{y}\right)} \sqrt{\psi_{x}^{2}+\psi_{y}^{2}}, \psi_{x}\right) W_{F T}\left(\psi_{x}, \psi_{y}\right) e^{j \psi_{y}\left(y_{0}-y_{p}\right)}
\end{aligned}
$$

a class of covariance operators $L_{\psi_{x}, \psi_{y}}^{\psi_{x}^{\prime}, \psi_{y}^{\prime}}$ can be obtained in the generalized form as reported in (35) at the bottom of the page. Consequently (35) allows to define different classes of intensity functions $I(x, y)$. In particular, using (20) the DASMUSIC model [11] is derived, while employing (14) the FTMUSIC reported in (22) is obtained again.

\section{NUMERICAL IMPLEMENTATION}

To test the performances of the presented reconstruction algorithms a stepped frequency radar operating in monostatic conditions is considered [4]. The radar system is composed of $N_{a}$ receiving-transmitting antennas located along the $x$-axis [see Fig. 5(a)] at a uniform distance $d_{a}>\frac{\lambda_{0_{\min }}}{4}$ from each other $\left(\lambda_{0_{\min }}\right.$ is the lowest free-space wavelength). All the antennas are considered fully matched when they operate in free space.

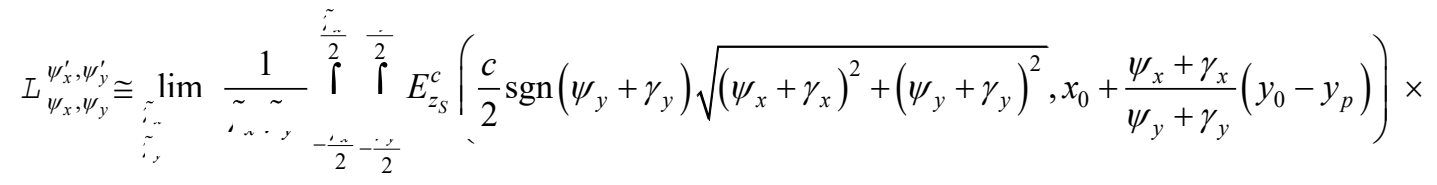

$$
\begin{aligned}
& \times E_{z_{S}}^{c^{*}}\left(\frac{c}{2} \operatorname{sgn}\left(\psi_{y}^{\prime}+\gamma_{y}\right) \sqrt{\left(\psi_{x}^{\prime}+\gamma_{x}\right)^{2}+\left(\psi_{y}^{\prime}+\gamma_{y}\right)^{2}}, x_{0}+\frac{\psi_{x}^{\prime}+\gamma_{x}}{\psi_{y}^{\prime}+\gamma_{y}}\left(y_{0}-y_{p}\right)\right) W^{\prime}\left(\psi_{y}+\gamma_{y}\right) W^{\prime^{*}}\left(\psi_{y}^{\prime}+\gamma_{y}\right) d \gamma_{x} d \gamma_{y}
\end{aligned}
$$

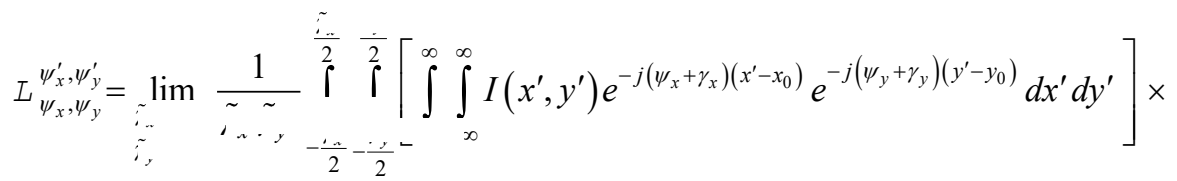

$$
\begin{aligned}
& \times\left[\int_{-\infty}^{\infty} \int_{-\infty}^{\infty} I\left(x^{\prime \prime}, y^{\prime \prime}\right) e^{-j\left(\psi_{x}^{\prime}+\gamma_{x}\right)\left(x^{\prime \prime}-x_{0}\right)} e^{-j\left(\psi_{y}^{\prime}+\gamma_{y}\right)\left(y^{\prime \prime}-y_{0}\right)} d x^{\prime \prime} d y^{\prime \prime}\right]^{*} d \gamma_{x} d \gamma_{y}
\end{aligned}
$$




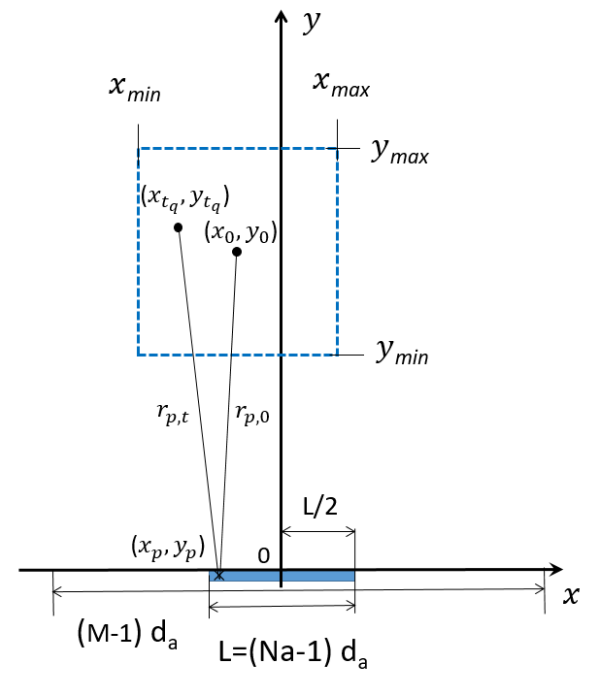

(a)

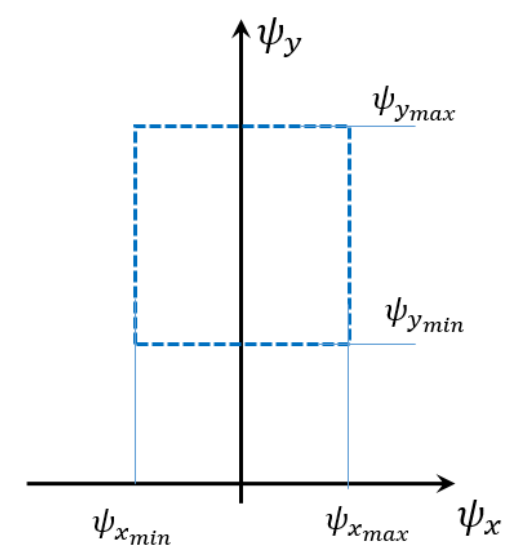

(b)

Fig. 5 Geometry of the scenario: x-y plane (a) and $\psi_{x}-\psi_{y}$ plane (b).

When scattering obstacles are present, non-vanishing antenna reflection coefficients are expected. In order to perform the spatial detection of the obstacles, the reflection coefficient of each of the $N_{a}$ antenna ports, evaluated at uniform spaced $N_{f}$ frequency values $f_{q}$ between $f_{\min }$ and $f_{\max }$ (frequency bandwidth $\left.B=f_{\max }-f_{\min }\right)$, is recorded in a $\underline{\Gamma}$ matrix, having $\Gamma_{p q}$ as a generic element, where $p$ is the antenna index ( $p=$ $\left.0, \ldots, N_{a}-1\right), q$ is the frequency index $\left(q=0, \ldots, N_{f}-1\right)$, and $f_{q}=f_{\min }+\frac{B}{N_{f}-1} q$. To evaluate the elements of the $\underline{\Gamma}$ matrix, only one transceiver at a time is considered active.

The reflection coefficient $\Gamma_{p q}$ takes the following form under the hypothesis of point-like targets located far from the antenna array

$\Gamma_{p q} \approx \sum_{t=1}^{N_{T}} \sigma_{t, q} \exp \left\{-j 4 \pi f_{q}\left(\frac{r_{p, t}}{c}+\tau_{D p}\right)\right\}+n_{p q}$

where $n_{p q}$ is a white zero mean Gaussian noise with $\sigma^{2}$ variance, $\sigma_{t, q}$ is a coefficient linked to the radar cross-section of the $t-t h$ target and on the electrical path of the RF signal.
In (36) $r_{p, t}$ is the distance between the antenna element located at $\left(x_{p}, y_{p}\right)$ and the $t-t h$ target located at $\left(x_{t_{q}}, y_{t_{q}}\right)$ given by [see Fig. 5(a)]:

$$
r_{p, t}=\sqrt{\left(x_{t_{q}}-x_{p}\right)^{2}+\left(y_{t_{q}}-y_{p}\right)^{2}}
$$

while $\tau_{D p}$ is the time delay due to the wave propagation between the excitation ports and the aperture plane of the $p-$ $t h$ antenna. To remove the error introduced by the propagation delay $\tau_{D_{p}}$ each measurement of the reflection coefficient $\Gamma_{p q}$ is analytically compensated as follows

$$
\Gamma_{p q}^{\prime}=\Gamma_{p q} \exp \left\{j 4 \pi f_{q} \tau_{D, p}\right\}
$$

\section{A. FT Based Algorithm}

This reconstruction technique is based on the Fourier Transform of the measured reflection coefficients $\Gamma_{p q}^{\prime}$ which are proportional to the array impinging electric field. With reference to Fig. 5(a), the numerical evaluation of (10) is expressed applying the FFT to all columns of $\Gamma_{p q}^{\prime}$ coefficients with a $M$ (even) zero padding, obtaining:

$$
\begin{array}{r}
\tilde{E}_{u q}=\left.\tilde{E}_{z_{S}}\left(\omega, \psi_{x}\right)\right|_{\omega=\omega_{q}}= \\
\psi_{x}=\psi_{x u}^{\prime} \\
=d_{a} e^{j 2 \pi \frac{u}{M d_{a}}\left(\frac{L}{2}+x_{0}\right)} \sum_{p=0}^{N_{a}-1} \Gamma_{p q}^{\prime} e^{-j \frac{2 \pi u p}{M}}
\end{array}
$$

where

$$
\psi_{x_{u}}^{\prime}=u \frac{2 \pi}{\mathrm{M} d_{a}} \quad u=-\frac{M}{2}, \ldots, \frac{M}{2}-1
$$

and

$$
\omega_{q}=2 \pi f_{q} \quad q=0, \ldots, N_{f}-1 .
$$

To express (39) in a regular rectangular grid $(u, v)$ associated with the variables $\psi_{x}^{\prime}, \psi_{y}^{\prime}$ [see Fig. 5(b)] a 3rd-order polynomial interpolation is used, thus obtaining

$$
\tilde{E}_{u v}^{\prime}=\left.\tilde{E}_{z_{S}}\left(\omega, \psi_{x}\right) e^{j \psi_{y_{v}}^{\prime}\left(y_{0}-y_{p}\right)}\right|_{\omega=\frac{c}{2} \sqrt{\psi_{x_{u}}^{\prime 2}+\psi_{y_{v}}^{\prime 2}}}
$$

with

$\psi_{y_{v}}^{\prime}=\psi_{y_{\min }}+v \frac{\psi_{y_{\max }}-\psi_{y_{\min }}}{\mathrm{N}-1} \quad v=0, \ldots, N-1$

where

$$
\begin{gathered}
\psi_{y_{\min }}=\frac{4 \pi f_{\min }}{c} \\
\psi_{y_{\max }}=\sqrt{\left(\frac{4 \pi f_{\max }}{c}\right)^{2}-\left(\frac{\pi}{d_{a}}\right)^{2}} .
\end{gathered}
$$


Finally, subdividing the ROI [dashed blue box in Fig. 5(a)] in a regular $\mathrm{N}_{x} \times \mathrm{N}_{y}$ square pixels having center coordinates

$x_{u^{\prime}}=x_{\min }+\frac{x_{\max }-x_{\min }}{N_{x}-1} u^{\prime} \quad u^{\prime}=0, \ldots, N_{x}-1$

$y_{v^{\prime}}=y_{\min }+\frac{y_{\max }-y_{\min }}{N_{y}-1} v^{\prime} \quad v^{\prime}=0, \ldots, N_{y}-1$

the FT intensity function (14) is evaluated as follows:

$$
\begin{aligned}
& I_{F T} u^{\prime} v^{\prime} \\
& =\frac{1}{M N} R e\left[\sum_{u=0}^{M-1} \sum_{v=0}^{N-1} \tilde{E}_{u v}^{\prime} e^{j \psi_{x_{u}}^{\prime}\left(x_{u^{\prime}}-x_{0}\right)} e^{j \psi_{y_{v}}^{\prime}\left(y_{v^{\prime}}-y_{0}\right)} W_{F T_{u v}}\right]
\end{aligned}
$$

with

$$
W_{F T_{u v}}=W_{F T}\left(\psi_{x_{u}}^{\prime}, \psi_{y_{v}}^{\prime}\right)
$$

where $\operatorname{Re}[\cdot]$ is the real part operator. Note that the normalization factor $M N$ in (48) has been added for computational convenience.

\section{B. FT-MUSIC Algorithm}

The MUSIC version of the FT algorithm is derived using a discrete form of the operator (22). To this end, the following flattener operator is introduced to simplify the implementation of the 2D numerical integration appearing in (22),

$$
\mathbf{Q}=\mathbf{F}_{l}(\underline{\boldsymbol{s}})=\left[S_{11} \ldots S_{M 1} S_{12} . . S_{M 2} . . S_{M N}\right]^{T}
$$

where $\underline{\boldsymbol{S}}$ is a matrix of size $M \times N$, @ is a vector having $M \cdot N$ elements, and $(\cdot)^{T}$ denotes the transpose operator. Then, defining the coefficients of the $M \times N$ matrix $\widetilde{\boldsymbol{E}}_{w}^{\prime}$ as

$$
\tilde{E}_{w_{u v}}^{\prime}=\tilde{E}_{u v}^{\prime} W_{F T}\left(\psi_{x_{u}}^{\prime}, \psi_{y_{v}}^{\prime}\right)
$$

the discrete form of the covariance operator (22) can be written in the following compact form:

$$
\underline{\boldsymbol{R}}=\frac{1}{N_{S}^{\prime}} \sum_{u^{\prime}=1}^{M-K+1} \sum_{v^{\prime}=1}^{N-L+1} \mathbf{F}_{l}\left(\underline{\boldsymbol{S}}_{u^{\prime} v^{\prime}}\right) \cdot \mathbf{F}_{l}\left(\underline{\boldsymbol{S}}_{u^{\prime} v^{\prime}}\right)^{H}
$$

where the $\underline{\boldsymbol{S}}_{\boldsymbol{u}^{\prime} v^{\prime}}$ are $K \times L$ submatrices extracted from the matrix $\widetilde{\boldsymbol{E}}_{w}^{\prime}(51)$, starting from $u^{\prime}$ and $v^{\prime}$ indexes which address the first element of the submatrix, and

$$
N_{S}^{\prime}=(\mathrm{M}-\mathrm{K}+1)(\mathrm{N}-\mathrm{L}+1) .
$$

In (52) $(\cdot)^{H}$ denotes the Hermitian transpose operator. In order to increase the numerical accuracy of (22), the improved numerical method proposed in [11] suitable for the computation of the covariance matrix $\underline{\mathbf{R}}$ is employed. As in [11], the covariance matrix $\underline{\mathbf{R}}$, having size $K L \times K L$, is computed combining together the submatrices $\underline{\boldsymbol{S}}_{u^{\prime} v^{\prime}}$ in the following manner

$$
\begin{aligned}
\underline{\boldsymbol{R}}= & \frac{1}{2 N_{S}^{\prime}} \sum_{u^{\prime}=1}^{M-K+1} \sum_{\substack{v^{\prime}=1 \\
N-L+1}}\left[\mathbf{F}_{l}\left(\underline{\boldsymbol{S}}_{u^{\prime} v^{\prime}}\right) \cdot \mathbf{F}_{l}\left(\underline{\boldsymbol{S}}_{u^{\prime} v^{\prime}}\right)^{H}+\right. \\
& \left.+\underline{\boldsymbol{J}} \cdot \mathbf{F}_{l}\left(\underline{\boldsymbol{S}}_{u^{\prime} v^{\prime}}\right)^{*} \cdot \mathbf{F}_{l}\left(\underline{\boldsymbol{S}}_{u^{\prime} v^{\prime}}\right)^{T} \cdot \underline{J}\right]
\end{aligned}
$$

where $\boldsymbol{J}$ is a square $K L \times K L$ matrix having a unitary value on the secondary diagonal, while $(\cdot)^{*}$ denotes the complex conjugate operator. Moreover, it is required that $K>N_{T}$ and $L>N_{T}$, where $N_{T}$ is the number of targets located in the ROI. Note that the covariance matrix $\underline{\mathbf{R}}$ is Hermitian, consequently its eigenvalues are real quantities, and its eigenvectors are orthogonal to each other.

According to the theory presented in the previous section, $\underline{\mathbf{R}}$ should have a number $N_{H}$ of the highest eigenvalues equal to the number $N_{T}$ of targets [see eq. (26)]. However, due to the numerical noise introduced by the discrete integral computation, additional not vanishing eigenvalues appear. This covariance matrix characteristic is exploited in the heuristic Akaike Information Criterion (AIC) [17] that is adopted to accurately evaluate the potential number of scatterers in the ROI. Using the $N_{H}$ normalized eigenvectors $\boldsymbol{w}_{u}$ of $\underline{\mathbf{R}}$ linked to the $N_{H}$ highest eigenvalues, the projector operator (27) is implemented as follows

$$
\underline{\boldsymbol{Q}}=\underline{\mathbf{1}}-\sum_{u=1}^{N_{H}} \boldsymbol{w}_{u} \cdot \boldsymbol{w}_{u}{ }^{H}
$$

where $\underline{\mathbf{1}}$ is the unitary dyad. Finally, the expressions (30) is evaluated to compute the FT-MUSIC intensity function suitable to identify the scattering targets. To do that, the intensity function $I_{\text {FTMUSIC }_{u^{\prime}, v^{\prime}}}$ is obtained using the following expression:

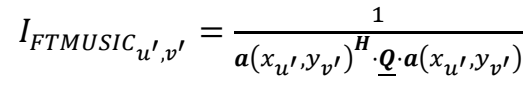

where the vector $\boldsymbol{a}(x, y)$ is given by (57) at the bottom of the page.

\section{MUSIC Algorithm}

The basic version of the MUSIC reconstruction technique (introduced as element-space MUSIC or ES-MUSIC in [11]) is implemented using the same procedure adopted for the FTMUSIC algorithm.

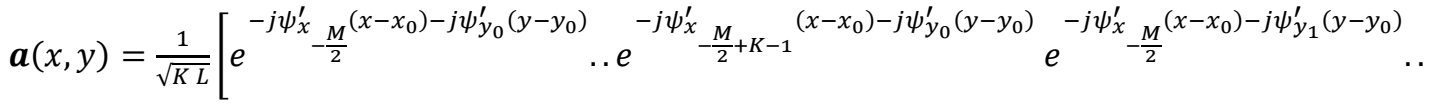

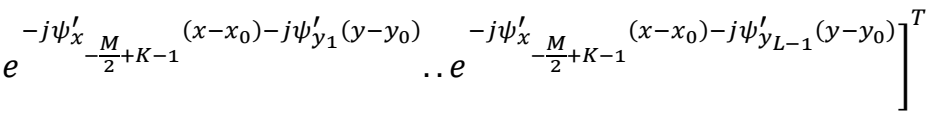


Since the MUSIC algorithm is based on the numerical evaluation of the covariance operator (31), depending on the reflection coefficients $\Gamma_{p q}^{\prime}$, a partial compensation of their rapid phase variation is first performed as follows,

$$
\bar{\Gamma}_{p q}^{\prime}=\Gamma_{p q}^{\prime} \exp \left\{\frac{j 4 \pi f_{q} r_{p, o}}{c}\right\}
$$

where $r_{p, o}$ identifies the distance between the $p$-th array antenna and the ROI center [see Fig. 5(a)]. Then, using (18), the coefficients $\bar{\Gamma}_{p q}^{\prime}$ are linked to the transverse $\psi_{x}^{\prime}$ and longitudinal $\psi_{y}^{\prime}$ wavenumbers, respectively, as follows

$$
\begin{gathered}
\bar{\Gamma}_{p q}^{\prime}=\bar{\Gamma}^{\prime}(\omega, x) \mid \begin{array}{l}
\omega=\omega_{q} \\
x=x_{p}
\end{array} \\
=\left.\overline{\bar{\Gamma}}^{\prime}\left(\psi_{x}^{\prime}, \psi_{y}^{\prime}\right)\right|_{\begin{array}{c}
\psi_{x}^{\prime}=-2 \\
\psi_{y}
\end{array}} \frac{\omega_{q}-x_{0}}{r_{p, o}}=\overline{\bar{\Gamma}}_{p q}^{\prime} . \\
\psi_{y=-2}^{\prime} \frac{\omega_{q}}{c} \frac{y_{p}-y_{0}}{r_{p, o}}
\end{gathered}
$$

Subsequently, a 3rd-order polynomial interpolation is performed to express $(59)$ by means of the $(u, v)$ indexes defined in (40) and (43), obtaining

$$
\overline{\bar{\Gamma}}_{u v}^{\prime \prime}=\overline{\bar{\Gamma}}^{\prime}\left(\psi_{x_{u}}^{\prime}, \psi_{y_{v}}^{\prime}\right) \begin{aligned}
& u=-\frac{M}{2}, \ldots, \frac{M}{2}-1 \\
& v=0, \ldots, N-1
\end{aligned} .
$$

Finally, the MUSIC intensity function $I_{M U S I C} C_{u^{\prime}, v^{\prime}}$ is computed by means of (57) using in this case $\overline{\bar{\Gamma}}_{u v}^{\prime \prime} W^{\prime}\left(\psi_{y_{v}}^{\prime}\right)$ instead of $\tilde{E}_{w_{u v}}^{\prime}$.

\section{DAS and DAS-MUSIC Algorithms}

As above discussed, two different DAS algorithms, namely basic DAS and DAS-MUSIC, can be implemented. The first algorithm, based on (20), computes the DAS intensity distribution inside the ROI using the following expression:

$$
I_{D A S_{u^{\prime} v^{\prime}}}=\frac{1}{N_{f} N_{a}} \operatorname{Re}\left[\sum_{q=0}^{N_{f^{-1}}} \sum_{p=0}^{N_{a}-1} \Gamma_{p q}^{\prime} W_{D A S_{p q}} e^{\left.j 2 \frac{\omega_{q}}{c} r_{p, u^{\prime} v^{\prime}}\right]}\right.
$$

where

$$
r_{p, u^{\prime} v^{\prime}}=\sqrt{\left(x_{u^{\prime}}-x_{p}\right)^{2}+\left(y_{v^{\prime}}-y_{p}\right)^{2}}
$$

and $W_{D A S_{p q}}$ is the weight function

$$
W_{D A S_{p q}}=W_{D A S}\left(\omega_{q}, x_{p}\right) .
$$

The DAS-MUSIC algorithm, as defined in (35), uses the DAS intensity function (61) as starting point for the target localization. A two-dimensional discrete Fourier transform of (61) is performed as,

$$
\begin{aligned}
& \mathcal{J}_{D S_{u^{\prime} v^{\prime}}}= \\
& =\frac{1}{N_{x} N_{y}} \sum_{u=0}^{N_{x}-1} \sum_{v^{\prime}=0}^{N_{y}-1} I_{D A S_{u^{\prime} v^{\prime}}} e^{-j \psi_{x_{u}}^{\prime}\left(x_{u^{\prime}}-x_{0}\right)} e^{-j \psi_{y_{v}}^{\prime}\left(y_{v^{\prime}}-y_{0}\right)}
\end{aligned}
$$

then, the DAS-MUSIC intensity function $I_{\text {DASMUSIC }} u_{u^{\prime}, v^{\prime}}$ is computed by means of the expression (57) considering, in this case, as input parameters $\mathcal{J}_{D S u_{u^{\prime} v^{\prime}}}$ instead of $\tilde{E}_{w_{u v}}^{\prime}$.

\section{E. Fermat's Principle and Normal Incidence Approximation}

To account for the refraction effect caused by a wall, the Fermat's principle of least time [1] has been implemented. Based on this principle, the electromagnetic path between the source and the scatterer, and vice versa, is the path that experiences the least propagation time. This means that the problem of finding $r_{p, u^{\prime} v^{\prime}}$ in (61) becomes the optimization problem of finding the wall coordinates points where the ray crosses the wall surfaces for the minimum travel time [11]. In many practical cases, an almost normal incidence of the field generated by the radar on the wall is experienced. In this case, the effect of the wall can be taken into account by simply adding a further delay term in (36) given by:

$$
\tau_{\mathrm{w}}=\frac{2 d}{c}\left(\sqrt{\varepsilon_{r}^{\prime}}-1\right)
$$

where $\mathrm{d}$ is the wall thickness and $\varepsilon_{r}^{\prime}$ it's the real relative dielectric permittivity. This delay time has to be compensated within the target identification procedures presented above.

The numerical procedure to rigorously evaluate the total electrical path length has been implemented in both DAS and DAS-MUSIC algorithms, while the approximated expression (65) has been applied with FT, MUSIC, and FT-MUSIC algorithms.

\section{EXPERIMENTAL SETUP REALIZATION}

\section{A. Vivaldi Antenna Performances}

A Vivaldi antenna has been designed using the full-wave software CST Microwave Studio and realized on a $0.813 \mathrm{~mm}$ (32 mills) RO4003C substrate. Eight samples have been realized at microwaves and electromagnetic compatibility laboratory of the Department of Information Engineering, Electronics and Telecommunications at Sapienza University of Rome by using a milling machine (T-tech, Quick Circuit 5000). The antenna return loss has been measured by using a vector analyzer (PNA Agilent E8363C). Fig. 6 shows the frequency behavior of the antenna input impedance in the 1-8 GHz frequency band where the antenna is well matched. As it can be seen, the agreement between measurements and numerical simulations is excellent. Similar results have been obtained for the other antennas. Then, the antenna radiation pattern has been measured inside the anechoic chamber available at Casaccia Enea Research Centre. Fig. 7 shows a comparison between the simulated and measured radiation patterns in the E-plane (a) and in the H-plane (b) at the central frequency of $2 \mathrm{GHz}$. Also in this case a good agreement between simulations and measurements can be observed. The antenna gain is $8.8 \mathrm{dBi}$, while the main beam aperture angle is $\theta_{\mathrm{E}}=47^{\circ}$ and $\theta_{\mathrm{H}}=76^{\circ}$ in the E- and in the H-planes, respectively. The radiation patterns have been also measured at $1 \mathrm{GHz}\left(\mathrm{Gain} 4.6 \mathrm{dBi}, \theta_{\mathrm{E}}=98^{\circ} \theta_{\mathrm{H}}\right.$ $\left.=150^{\circ}\right)$ and $3 \mathrm{GHz}\left(\right.$ Gain $\left.9.9 \mathrm{dBi}, \theta_{\mathrm{E}}=41^{\circ}, \theta_{\mathrm{H}}=50^{\circ}\right)$. 


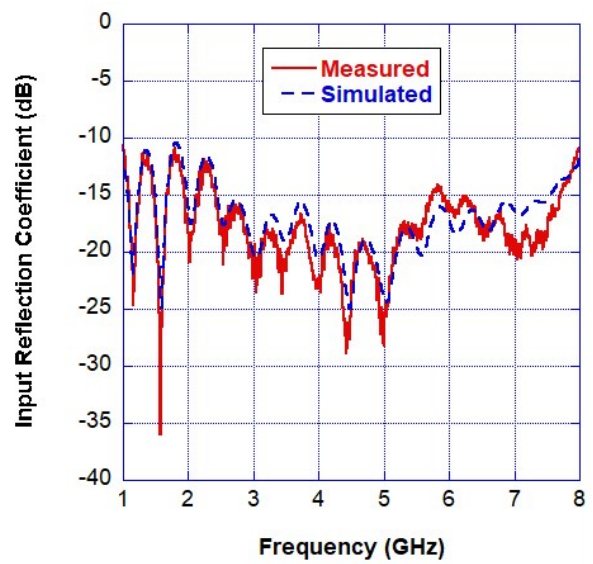

Fig. 6. Comparison between measured and simulated antenna input reflection coefficient of one of the realized antennas.

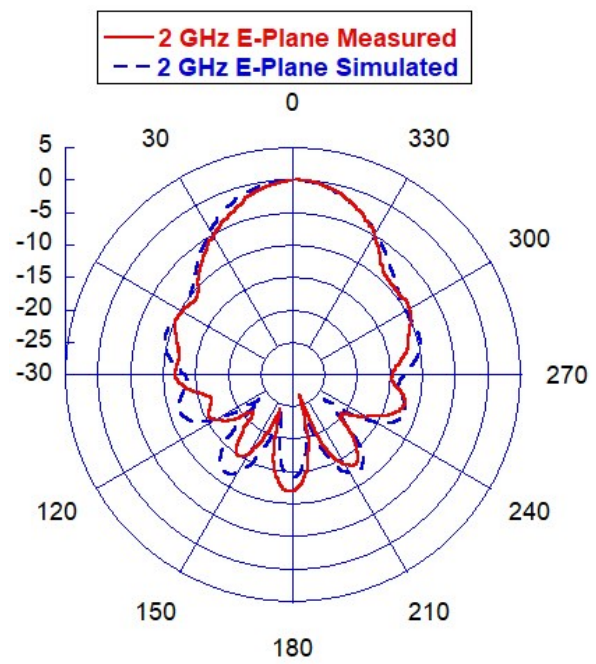

(a)

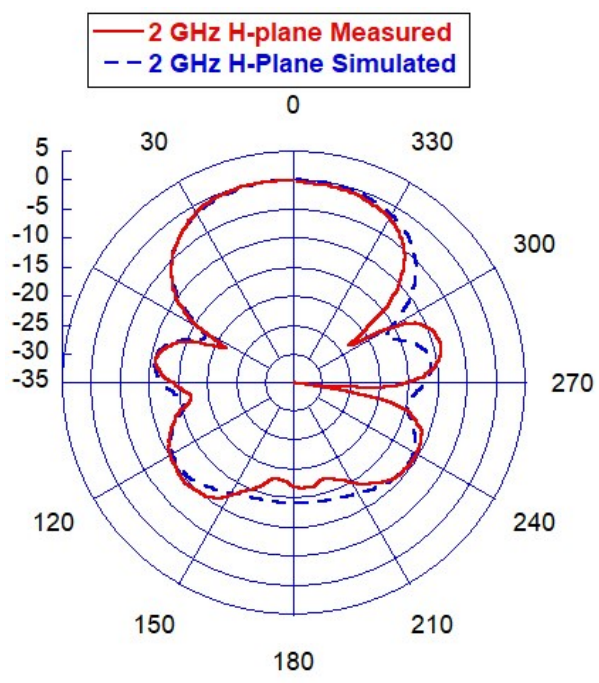

(b)

Fig. 7. Comparison between the simulated and measured radiation pattern in (a) E- and (b) H-planes. An excellent agreement between numerical results and measurements can be observed.

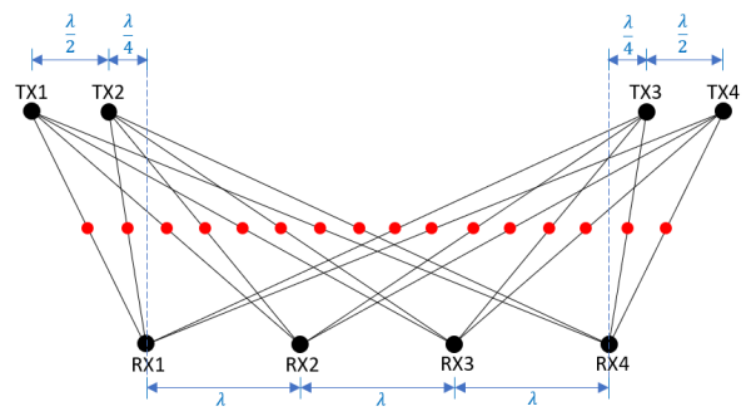

Fig. 8. Spatial positioning of the transmitting and receiving antennas.

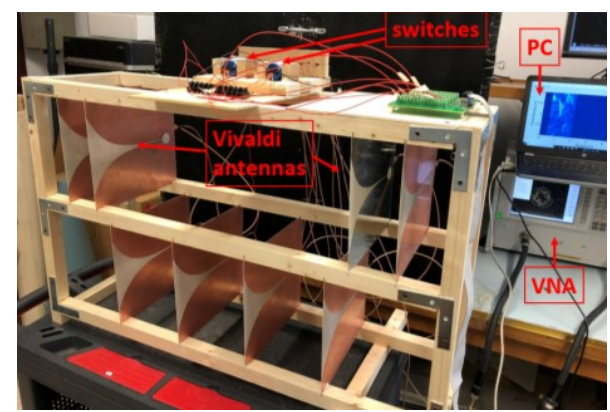

Fig. 9. Radar experimental setup. The eight Vivaldi antennas, together with the switches and the Vector analyzer are visible in the figure.

\section{B. Radar Structure}

Fig. 1 shows the schematic of the stepped-frequency radar structure. The realized system utilizes a vector network analyzer (Agilent M5230A), with a $110 \mathrm{~dB}$ dynamic range, to measure the $S_{21}$ scattering parameters between the transmitting and the receiving section. A $0 \mathrm{dBm}$ output power is usually employed. For measurements in the presence of a wall a $20 \mathrm{~dB}$ power amplifier (PA) (HP 8349A) has been added in the transmitting channel. A couple of SP4T switches (Pastrernak PE71S6088) selects sequentially a transmitting and a receiving Vivaldi antenna. The spatial positioning of the transmitting and receiving antennas is chosen as indicated in Fig. 8. In this way, a series of equidistant equivalent antennas separated by a quarter of the free-space wavelength is realized. Following the spatial positioning indicated in Fig. 8 eight antennas have been housed in a wood box (see Fig. 9). The overall system is controlled by a LabVIEW program that, driving the TTL ports of the switches sequentially selects a couple of transmitting and receiving antennas and through the VNA GPIB port acquires the scattering parameter data over 500 frequency points. Once the signals from the sixteen positions have been acquired the data are stored in a 500x16 matrix and saved on the computer memory. The data are then read by Matlab ${ }^{\mathrm{TM}}$ programs where the reconstruction techniques are implemented. The proposed radar system acquires the 500x16 matrix complex elements in about $20 \mathrm{~s}$. To make the system compatible with the time scales of subject's movements, this time could be reduced, for example, by reducing the number of points acquired or by using a dedicated field-programmable gate array (FPGA) for the control of switches and for data acquisition. 


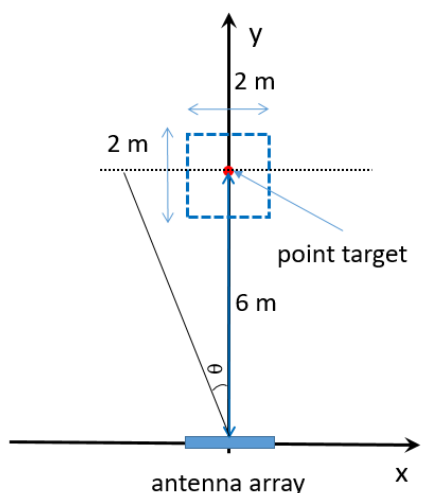

Fig. 10. Geometry of the simulated scenario with antenna array and single point scatterer.

\section{NUMERICAL AND EXPERIMENTAL RESULTS}

\section{A. Image Reconstructions With Analytical Data}

In order to test the DAS, FT, MUSIC, DAS-MUSIC and FTMUSIC algorithms a single point scatterer located six meters far from the antenna array plane has been considered (see Fig. 10). The investigated scenario consists of a $2 \times 2 \mathrm{~m}$ square region subdivided in square cells with $1 \mathrm{~cm}$ side. $N_{a}=32$ antennas with $\mathrm{d}_{\mathrm{a}}=\lambda / 2$ (at $\mathrm{f}=2 \mathrm{GHz}$ ) spacing have been taken into account and (36) has been used to evaluate the scattering parameters at 500 frequency points between 1 and $3 \mathrm{GHz}$ (frequency band $\mathrm{Bw}$ $=2 \mathrm{GHz}$, frequency step $\Delta \mathrm{f}=4 \mathrm{MHz}$ ). A Gaussian noise with variance equal to $\sigma^{2}=0.1$ has been added to all the scattering parameters. For the considered scenario the radar theoretical resolution in range is $\delta \mathrm{r}=\mathrm{c} / 2 \mathrm{~B}=7.5 \mathrm{~cm}$, the angular resolution is $\delta \varphi=1 /\left(N_{a}-1\right)=0.032 \mathrm{rad}\left(1.85^{\circ}\right)$, and the unambiguous range is $R_{\max }=\mathrm{c} / 2 \Delta \mathrm{f}=37.5 \mathrm{~m}$.

Fig. 11 shows the results of the simulations performed by using the five considered techniques. In the figure, cuts along the range (a) and cross-range (b) directions performed on the image reconstructions based on the considered techniques have been reported for comparison purpose. In particular, the normalized intensity function in $\mathrm{dB}$ versus position is reported. From Fig. 11 it results that the half power beam width (HPBW) for both DAS and FT is of $7 \mathrm{~cm}$ in range [Fig. 11(a)] and $17 \mathrm{~cm}$ in cross-range [Fig. 11(b)] very close to the 7.5 and $19 \mathrm{~cm}$ theoretical resolutions achievable by the array.

The MUSIC algorithm $\left(\mathrm{K}=\mathrm{L}=10, \mathrm{~N}_{\mathrm{H}}=1\right)$ presents a HPBW equal to $1 \mathrm{~cm}$ and $2 \mathrm{~cm}$ in range and cross range, respectively, for the DAS-MUSIC $\left(\mathrm{K}=\mathrm{L}=10, \mathrm{~N}_{\mathrm{H}}=1\right)$ and the FT-MUSIC $\left(\mathrm{K}=\mathrm{L}=10, \mathrm{~N}_{\mathrm{H}}=1\right)$ the Range and Cross-range $\mathrm{HPBW}$ are $1.5 \mathrm{~cm}$ and $1.5 \mathrm{~cm}$, respectively. Fig. 11 shows the better performances of MUSIC, DAS-MUSIC and FT-MUSIC algorithms with respect to DAS and $\mathrm{FT}$ in particular along the cross-range direction. In the case of a scenario with strong attenuations as those produced by walls or by the presence of weak or distant scatterers, there will be a reduction in the signal to noise ratio which could influence the reconstruction techniques. In order to test the algorithms' ability to operate in these conditions, the simulations were repeated by increasing the noise variance by a factor of $10\left(\sigma^{2}=1\right)$. The obtained reconstructions (not reported for the sake of brevity) were very similar to those in

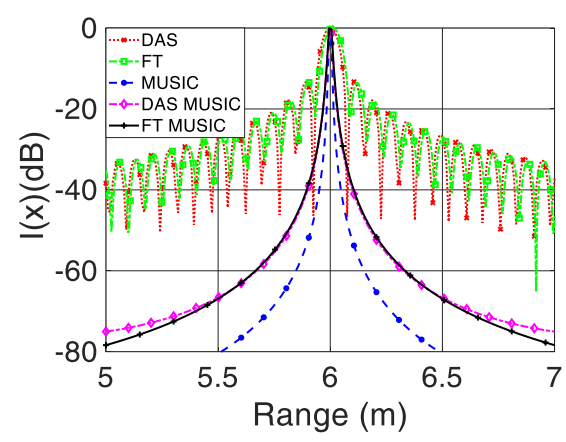

(a)

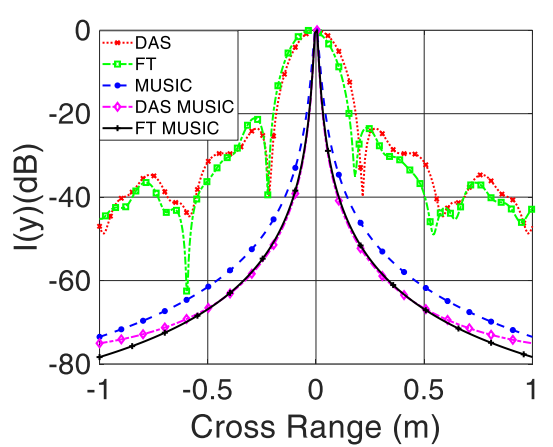

(b)

Fig. 11. Comparison between the five considered techniques. The normalized pixel intensity in $\mathrm{dB}$ is reported as a function of the position. Image cuts along the range (a) and cross-range (b) directions.

Fig. 11. In particular, the DAS, FT and DAS-MUSIC algorithms maintained the same HPBW as in the case $\sigma^{2}=0.1$ while the HPBW increased to $1.5 \mathrm{~cm}$ and $4.5 \mathrm{~cm}$ in range and cross range, respectively for the MUSIC algorithm and $2.5 \mathrm{~cm}$ in range and cross range for the FT-MUSIC algorithm.

The elaboration times (ET) required for data processing by the presented algorithms (Matlab program running on Pentium i7 processor) are: DAS: $30 \mathrm{~s}, \mathrm{FT}: 1 \mathrm{~s}$, MUSIC: $36 \mathrm{~s}$, DAS-MUSIC: 197 s, FT-MUSIC: 36 s. The elaboration time of the DAS algorithm is essentially related to the numerical solution of (61). Concerning the FT algorithm, the ET is due to the two FFTs appearing in (39) and (48). With reference to the MUSIC, the two cycles in (54) and (56) are the main responsible of the execution time. Similarly, for the FT-MUSIC where the initial FFT takes a negligible time. Finally, the DAS-MUSIC requiring three steps combines the times of DAS and MUSIC to the time necessary to compute (64). In conclusion, The FT algorithm presents the best ET performance followed by the MUSIC and FT-MUSIC ones. Additional simulations were performed up to a distance of about $12 \mathrm{~m}$ obtaining results (not reported for the sake of brevity) for the range cut very similar to those reported in Fig. 11(a). Concerning the cross-range cuts, the MUSIC, DAS-MUSIC and FT MUSIC present the same behavior of Fig. 11(b) while for DAS and FT the HPBW increases up to about $35 \mathrm{~cm}$. These last results are in agreement with the previous reported range and angular resolution definitions that foresees a range resolution constant with the distance while the cross-range resolution worsens when the distance increases. 


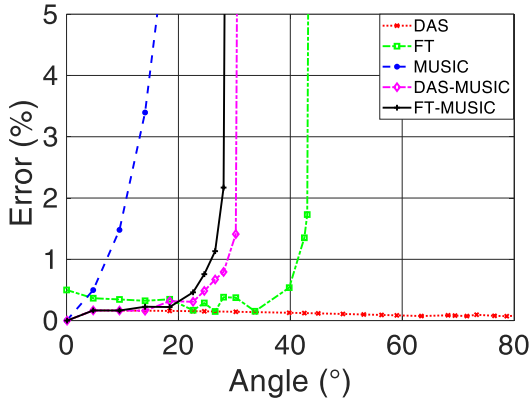

(a)

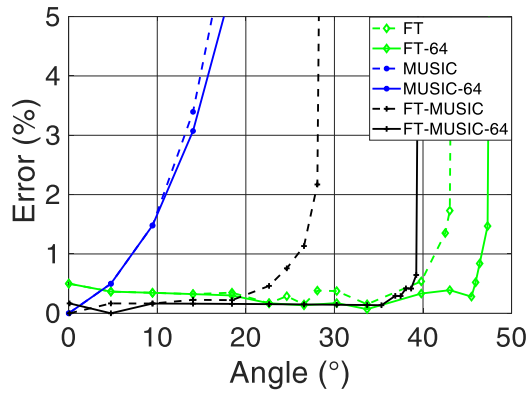

(b)

Fig. 12. Error comparison between the five considered techniques. The percentage error on the estimated distance is reported as a function of the angle for 32 antennas (a) and 64 antennas (b). In (b) the results for 32 antennas are also reported (dashed lines) for comparison purposes.

To evaluate the accuracy of the investigated techniques in assessing the target position, the target has been moved in crossrange (dashed black line in Fig. 10) at different angles with respect to the antenna center ( $\theta$ in Fig. 10). Fig. 12 shows the percentage error on the estimated distance with respect to the real one obtained for the five considered algorithms. Fig. 12(a) shows that when 32 antennas are used the best responses are given by the DAS algorithm that is able to recognize with a very high numerical accuracy the obstacle position up to angles greater than $80^{\circ}$. The FT is less accurate and is reliable up to about $40^{\circ}$. DAS-MUSIC and FT-MUSIC have similar behaviors and fail in the reconstruction after about $30^{\circ}$. The worst response is that provided by MUSIC algorithm which stops at about $20^{\circ}$. The MUSIC algorithm presents an error rapidly growing with the angle. This behavior is due to the approximation (17) on which this algorithm is based. This approximation fails when the target moves far from the antenna array center. Concerning the FT, MUSIC and FT-MUSIC algorithms, simulations have been repeated for a 64 antenna elements configuration whose results are reported in Fig. 12(b) (dashed lines) together with those achieved with 32 antennas (continuous lines). The increased antenna array dimensions determine an increase in the maximum detectable angle for the FT-MUSIC and to a lesser extent for the MUSIC and DASMUSIC algorithms.

\section{B. Reconstructions With Measured Data}

The realized radar system (see Fig. 9) has been used to fill the $\Gamma$ matrix used in the reconstruction algorithms. As evidenced in (36), to correctly evaluate this matrix it is

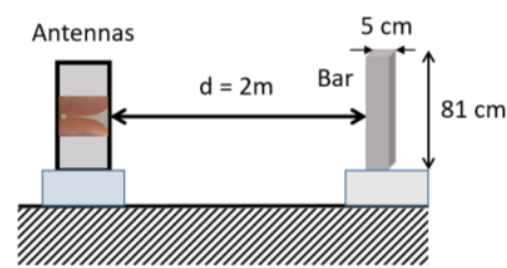

Fig. 13. Metallic bar placed $5 \mathrm{~m}$ far from the antennas.

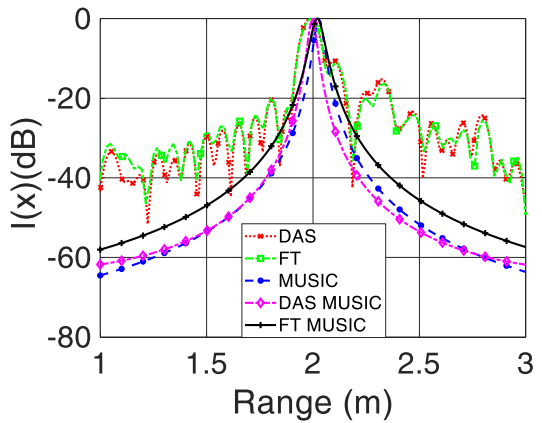

(a)

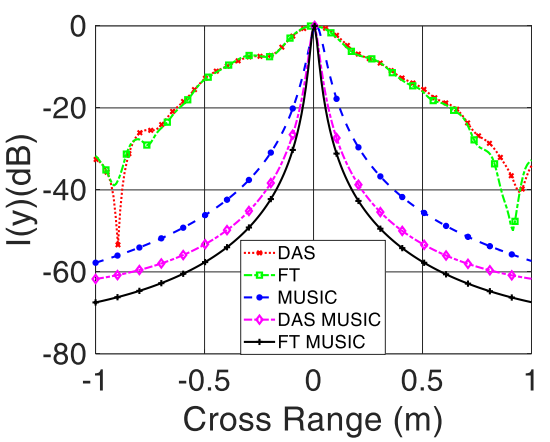

(b)

Fig. 14. Comparison between the five considered techniques. The normalized pixel intensity in $\mathrm{dB}$ is reported as a function of the position. Image cuts along the range (a) and cross-range (b) directions.

necessary to estimate the time delay $\left(\tau_{\mathrm{D}}\right)$ between the VNA calibrated ports and the antenna aperture plane. This delay can be evaluated simply by placing a transmitting and receiving antenna, connected to the VNA, facing each other. By analyzing the time domain pulse response on the VNA, it is possible to determine the signal delay time between the two calibrated ports. This measurement must be repeated for different distances and finally, through a linear regression formula, the intercept point of the resulting fitting line with the time axis is the searched delay time $\tau_{\mathrm{D}}$. Using this technique, a value of about $\tau_{\mathrm{D}}=2.32 \mathrm{~ns}$ has been found for all the array antennas.

Measurements have been performed in a hall of the EneaCasaccia research center, Rome, Italy. To reduce the effect of environmental clutter, and in particular the masking effect of the wall, the reflection coefficients used in the considered reconstruction algorithms were evaluated through the mathematical difference between those measured in presence and absence of obstacles. 


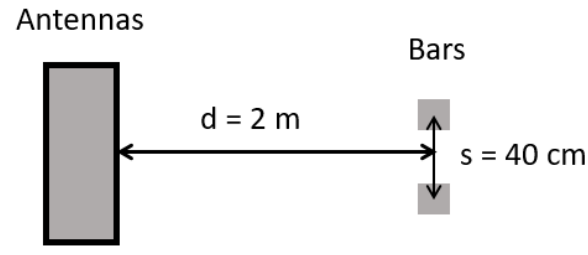

Fig. 15. Metallic bar placed $5 \mathrm{~m}$ far from the antennas (free-space case). Top view.

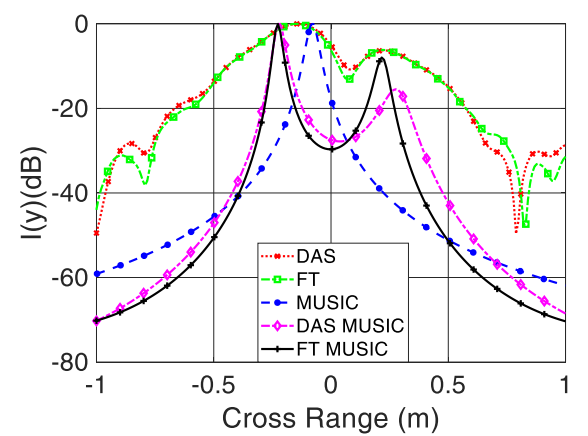

Fig. 16. Comparison between the five considered techniques. The normalized pixel intensity in $\mathrm{dB}$ is reported as a function of the position. Image cuts along cross range.

This technique, called background subtraction, does not eliminate the effect of multiple reflections such as those between scatterers or between the wall and the scatterers which, however, in practical cases, do not produce significant artifacts. Even though the background subtraction applied to realistic operative scenarios does not allow to identify the presence of fixed targets, it remains effective to detect moving targets. In fact, by employing the last acquisition as a background, it allows to follow the movement of a target over time. In the case of static targets, various heuristic approaches have been reported in the literature to mitigate wall clutter effects without knowledge of the background scene [23]-[25]. Among the others, statistical methods [23], spatial filtering [24], modeling and subtraction of the wall returns [25] have been proposed. Since the present work is mainly focused on carrying out an accurate comparison among various reconstruction techniques, the background subtraction technique has been chosen as the most suitable for this purpose. In addition, it can be usefully employed in a lab where measurements with and without targets can be easily performed. The first investigated scenario was constituted by a metallic bar (height $=81 \mathrm{~cm}$ ) with a square section (side $=5 \mathrm{~cm}$ ) placed $2 \mathrm{~m}$ far from the antennas (see Fig 13). Fig. 14 shows a comparison among the five considered techniques applied to a ROI of $2 \mathrm{~m} \times 2 \mathrm{~m}$ around the target. In particular, range cut, Fig. 14(a), and cross-range cut, Fig. 14(b), of the achieved images are reported. DAS and FT algorithms show comparable results, while MUSIC $\left(\mathrm{K}=\mathrm{L}=10, \mathrm{~N}_{\mathrm{H}}=1\right)$, DAS-MUSIC $\left(\mathrm{K}=\mathrm{L}=10, \mathrm{~N}_{\mathrm{H}}=1\right)$ and FT-MUSIC $(\mathrm{K}=\mathrm{L}=10$, $\mathrm{N}_{\mathrm{H}}=1$ ) algorithms have better performances. In particular, the super resolution properties are well evidenced in the crossrange cuts.

In a second experimental scenario two metallic bars (height $=81 \mathrm{~cm})$ with a square section $($ side $=5 \mathrm{~cm})$ located at a distance $\mathrm{s}=40 \mathrm{~cm}$ have been placed $2 \mathrm{~m}$ far from the antennas

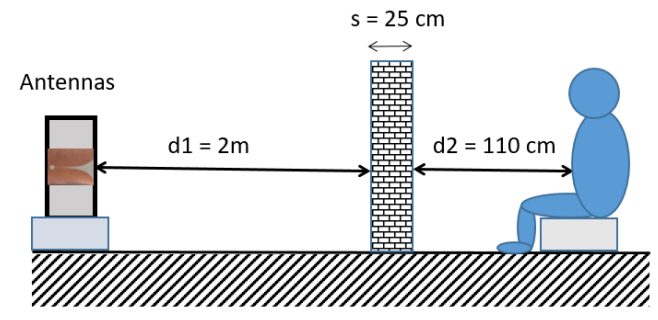

Fig. 17. Human subject placed $110 \mathrm{~cm}$ behind a $25 \mathrm{~cm}$ thick tuff wall located 2 $\mathrm{m}$ far from the antenna plane.

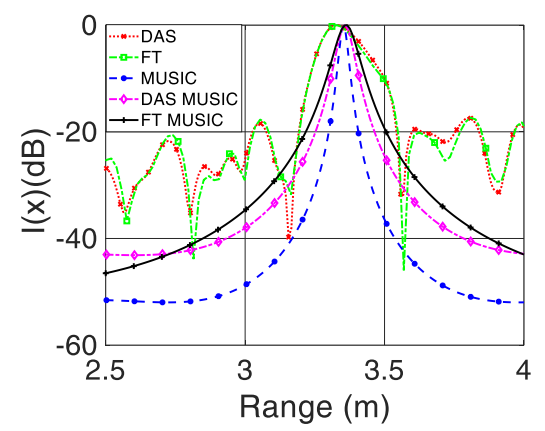

(a)

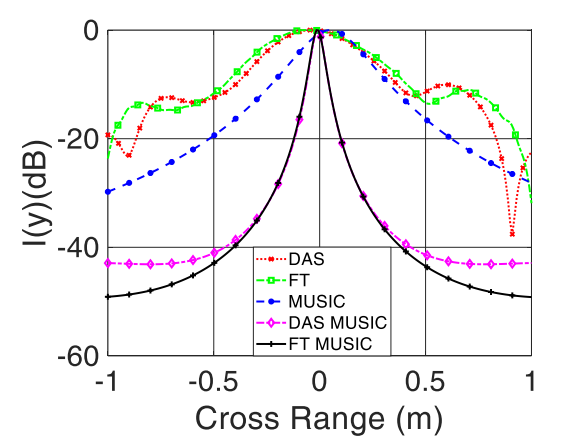

(b)

Fig. 18. Comparison between DAS and DAS-MUSIC algorithm using the Fermat principle and MUSIC, DAS-MUSIC, and FT-MUSIC algorithms using the normal incidence approximation.

(see Fig. 15). The considered ROI was $2 \times 2 \mathrm{~m}$ corresponding to $200 \times 200$ pixels. Fig. 16 shows a comparison among the five considered techniques along a cross-range cut of the achieved images. The DAS and FT algorithms show comparable results with two peaks of different amplitude due to the differences in the geometry and positions of the two bars, the FT-MUSIC $\left(\mathrm{K}=\mathrm{L}=10, \quad \mathrm{~N}_{\mathrm{H}}=2\right) \quad$ and DAS-MUSIC $\quad\left(\mathrm{K}=\mathrm{L}=10, \quad \mathrm{~N}_{\mathrm{H}}=2\right)$ algorithms were able to better recognize the two bars, while the MUSIC $\left(K=L=10, N_{H}=2\right)$ fails in separating the two peaks.

In this case, the poor performance of the MUSIC algorithm compared to DAS-MUSIC and FT-MUSIC ones is probably due to a greater sensitivity of this algorithm to measurement errors. In fact, the two hybrid techniques, being based on the signals provided by the DAS and the FT algorithms manage to operate even in conditions of low signal-to-noise ratio [11].

In the third experimental scenario a human subject has been placed $110 \mathrm{~cm}$ behind a $25 \mathrm{~cm}$ thick tuff wall (width $=180 \mathrm{~cm}$, 
height $=120 \mathrm{~cm}$ ) located $2 \mathrm{~m}$ far from the antenna plane as shown in Fig. 17. In this case a $20 \mathrm{~dB}$ amplifier has been added to the measurement systems (PA in Fig. 1) and its group delay has been measured obtaining $\tau_{\mathrm{a}}=5$ ns. For the relative permittivity of the tuff wall the value of 3.5 has been used as suggested in [26]. Fig 18 shows the plot for the DAS ad DASMUSIC $\left(\mathrm{K}=\mathrm{L}=10, \mathrm{~N}_{\mathrm{H}}=1\right)$ algorithms achieved by applying the Fermat principle and the plot for the FT, MUSIC $\left(\mathrm{K}=\mathrm{L}=20, \mathrm{~N}_{\mathrm{H}}\right.$ $=1)$, and FT-MUSIC $\left(\mathrm{K}=\mathrm{L}=20, \mathrm{~N}_{\mathrm{H}}=1\right)$ achieved by applying the normal incidence approximation. In all cases, the range position of the target (located at about $3.35 \mathrm{~m}$ ) is correctly reconstructed.

The last considered scenario consists of two metal panels of different sizes placed behind the wall (see Fig. 19). The reconstruction obtained with the FT algorithm is shown in Fig. 20(a). A similar result (not reported for the sake of brevity) was obtained with the DAS algorithm. In this case, the MUSIC, DAS-MUSIC and FT-MUSIC algorithms are unable to reconstruct the scenario correctly. In particular, they evidence only one peak in correspondence of the largest panel. This behavior is a consequence of the large cross distance of the scatterers with respect to ROI center. This situation causes a loss of approximations validity on which the proposed algorithms are based. To overcome this drawback, these three reconstruction techniques were applied by dividing the domain under analysis into four new ROIs formed by equal and adjacent quadrants of $2 \times 2 \mathrm{~m}$ (see Fig. 19). The results obtained with the DAS-MUSIC $\left(\mathrm{K}=\mathrm{L}=20, \mathrm{~N}_{\mathrm{H}}=10\right)$ and FT-MUSIC $\left(\mathrm{K}=\mathrm{L}=20, \mathrm{~N}_{\mathrm{H}}=10\right)$ algorithms are shown in Fig. 20(b) and 20 (c), respectively. The figures show that, by adopting this strategy (ROIs of smaller dimensions), the two algorithms are able to reconstruct the position of the two scatterers. In particular, the FT-MUSIC algorithm highlights two scattering centers located along the panels edges with a great accuracy. Notice that, the MUSIC algorithm (not reported for the sake of brevity) was not able to detect the images of the two scatterers even with a subdivision of the ROI region.

Table I at the bottom of the page reports a comparison among the Radar system proposed in this work and those existing in the literature. In particular, in the table, the Max Range column refers to measurements reported in the cited articles and does not represent the limit of the aforementioned systems which is also dependent on the type of wall and scatterer considered. For all the systems the range and cross range resolutions are very close to the theoretical ones. Finally, it is important to note that the Radar system presented in this work has been used to experimentally test the presented techniques. However, thinking of its practical use in realistic scenarios, the transmitted power could be increased up to $46 \mathrm{dBm}$ (maximum power tolerated by the switches) which would give rise to a considerable increase in the maximum investigable range.

\section{CONCLUSIONS}

The power budget for a TWRI scenario, evaluated through the radar equation taking into account the frequency behavior of the wall permittivity and of the scatterer radar cross section, has been presented. This analysis suggested the $1-3 \mathrm{GHz}$ frequency band as the most suitable for TWRI applications.

To test the performances of some algorithms useful for locating targets placed beyond a wall, a fast low cost TWRI system has been realized. The adopted hardware, based on an array of 16 equivalent elements operating between 1 and $3 \mathrm{GHz}$, is controlled by a LabVIEW program. The system employs a stepped-frequency radar architecture realized with a vector network analyzer, a couple of switches and an array of high gain wideband Vivaldi antennas. To limit the errors caused by the transit time of the RF signal in the Tx and Rx chains and by the environmental clutter, suitable calibration techniques have been implemented by means of preliminary measurements and adhoc data processing.

Then, an original theoretical modeling for a class of TWRI techniques has been introduced. In particular, the equations of FT and DAS algorithms have been derived using as basic function the cross range Fourier Transform of the scattered field. Moreover, a covariance operator of the same function is used to obtain the equations of DAS-MUSIC and MUSIC algorithms. The same operator has also been used to derive a novel algorithm, namely the hybrid FT-MUSIC. The above techniques have been implemented to operate on both analytical and experimental data and tested numerically and experimentally on scatterers consisting of metal objects and human subjects.

TABLE I

Comparison Among the Proposed RADAR System AND Those Existing IN the Literature

\begin{tabular}{|c|c|c|c|c|c|c|}
\hline & $\begin{array}{l}\text { Type of } \\
\text { Radar }\end{array}$ & $\begin{array}{l}\text { Frequency } \\
\text { Range }\end{array}$ & $\begin{array}{l}\text { Peak } \\
\text { Power }\end{array}$ & Antennas & Algorithms & $\begin{array}{c}\text { Max } \\
\text { Range }\end{array}$ \\
\hline This work & SFR & $1-3 \mathrm{GHz}$ & $20 \mathrm{dBm}$ & $\begin{array}{l}4 \text { TX and } 4 \text { RX } \\
\text { Vivaldi }\end{array}$ & $\begin{array}{l}\text { DAS, FT, MUSIC, DAS } \\
\text { MUSIC, FT-MUSIC }\end{array}$ & $5.35 \mathrm{~m}$ \\
\hline Lincoln Laboratory [3] & FMCW & $\begin{array}{l}1.926 \mathrm{GHz}- \\
4.069 \mathrm{GHz}\end{array}$ & $10 \mathrm{dBm}$ & $\begin{array}{l}1 \mathrm{TX} \text { and } 1 \mathrm{RX} \\
\text { movable slot } \\
\text { antennas }\end{array}$ & Range migration & $12 \mathrm{~m}$ \\
\hline Lincoln Laboratory [4] & FMCW & $2-4 \mathrm{GHz}$ & $30 \mathrm{dBm}$ & $\begin{array}{l}13 \mathrm{TX} \text { and } 8 \mathrm{RX} \\
\text { Vivaldi }\end{array}$ & Range migration & $20 \mathrm{~m}$ \\
\hline $\begin{array}{l}\text { US Army Research } \\
\text { laboratory [7]-[8] }\end{array}$ & UWB & $0.3-3 \mathrm{GHz}$ & $37 \mathrm{dBm}$ & $\begin{array}{l}2 \text { TEM horn TX } 16 \\
\text { Vivaldi RX }\end{array}$ & Back projection & $25 \mathrm{~m}$ \\
\hline $\begin{array}{l}\text { University of Tennessee } \\
\text { [9] }\end{array}$ & UWB & 2-6 GHz & $25 \mathrm{dBm}$ & $\begin{array}{l}1 \mathrm{TX} \text { and } 8 \mathrm{RX} \\
\text { Vivaldi }\end{array}$ & Back projection & $15 \mathrm{~m}$ \\
\hline $\begin{array}{l}\text { Villanova University [12]- } \\
\text { [14] }\end{array}$ & SFR & $1-3 \mathrm{GHz}$ & $10 \mathrm{dBm}$ & $\begin{array}{l}2 \mathrm{TX} \text { and } 1 \mathrm{RX} \\
\text { movable Horn }\end{array}$ & Compressive sensing & $5 \mathrm{~m}$ \\
\hline
\end{tabular}




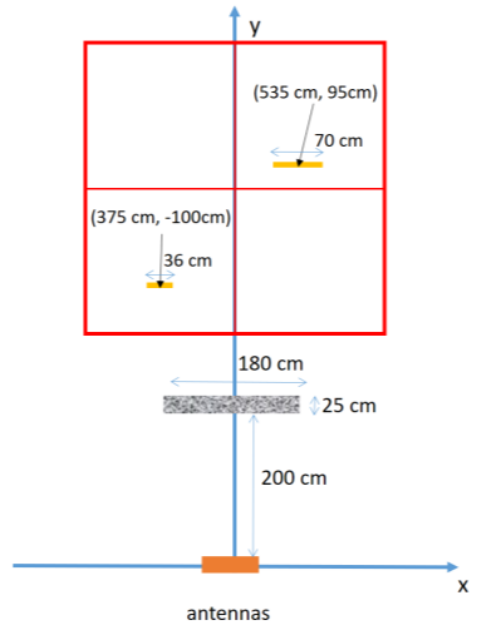

Fig. 19. Two metal panels behind a $25 \mathrm{~cm}$ thick tuff wall located $2 \mathrm{~m}$ far from the antenna plane. Top view.

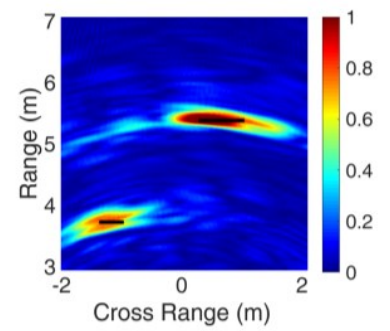

(a)

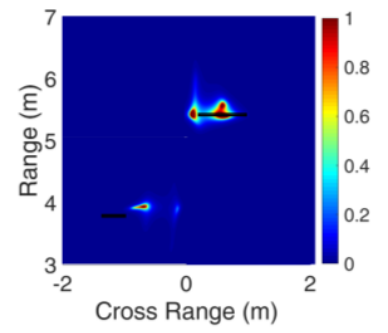

(b)

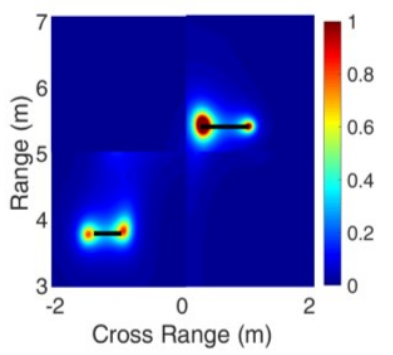

(c)

Fig. 20. Normalized pixel intensity obtained with FT algorithm (a), DASMUSIC algorithm (b) and FT-MUSIC algorithm (c). Domain subdivided as indicated in Fig. 19.

Numerical results have shown that the DAS algorithm presents a HPBW, both in range and cross range, in agreement with theoretical expectations and a wide field of view. Computational times of this technique are high especially for large ROI. The HPBW of the FT technique is very similar to the DAS one. With respect to DAS, this technique has a smaller field of view but extremely low elaboration times.

The proposed FT-MUSIC algorithm has evidenced characteristics similar to those of the other super resolution techniques with significantly lower computation times. In particular, this last property is due to the fact that the FTMUSIC algorithm employs the Fast Fourier transform routines that exhibit very low execution times as confirmed by the performance of the FT algorithm.

Using the proposed TRWI system, it was possible to identify with high accuracy human subjects and multiple reflecting targets of different sizes located beyond a tuff wall up to a maximum distance of about $5 \mathrm{~m}$. This range limit is linked to the minimum signal-to-noise ratio that can be handled by the implemented system. Anyway, given the modular structure of the proposed system, it is possible to easily extend its performance in terms of distance and spatial resolution by increasing the gain of RF amplifiers and the number of radiating elements.

\section{REFERENCES}

[1] M. G. Amin, "Through the wall radar imaging", CRC Press, Boca Raton, FL, USA, 2011.

[2] G. Wang, C. Gu, T. Inoue, and C. Li, "A hybrid FMCW-interferometry radar for indoor precise positioning and versatile life activity monitoring," IEEE Trans. Microw. Theory Techn., vol. 62, no 11, pp. 2812-2822, Nov. 2014.

[3] G. L. Charvat, L. C. Kempel, E. J. Rothwell, C. M. Coleman, and E. L. Mokole, "A through-dielectric radar imaging system," IEEE Trans. Antennas Propag., vol. 58, no 8, pp. 2594-2603, Aug. 2010.

[4] J. E. Peabody, G. L. Charvat, J. Goodwin, and M. Tobias, "Through-wall imaging radar," Lincoln Laboratory Journal, vol. 9, no 1, pp. 62-72, 2012.

[5] F. Adib, C.Y. Hsu, H. Mao, D. Katabi, and F. Durand, "Capturing the human figure through a wall," ACM Trans. Graph., vol. 34, no 6, Nov. 2015.

[6] F. Fioranelli, S. Salous, I. Ndip, and X. Raimundo, "Through-the-wall detection with gated FMCW signals using optimized patch-like and Vivaldi antennas," IEEE Trans. Antennas Propag., vol. 63, no 5, pp. 1106-1117, Mar. 2015.

[7] M. A. Ressler, L. H. Nguyen, F. J. Koenig, and G. Smith, "Synchronous impulse reconstruction (SIRE) radar sensor for autonomous navigation," US Army Research Laboratory, Adelphi, MD, Nov. 2006.

[8] C. Le, T. Dogaru, L. Nguyen, and M. A. Ressler, "Ultrawideband (UWB) radar imaging of building interior: measurement and prediction," IEEE Trans. Geosci. Remote Sens., vol. 47, no 5, pp. 1409-1420, May 2009.

[9] Y. Wang, and A.E. Fathy, "Advanced system level simulation platform for three-dimensional UWB through-wall imaging SAR using timedomain approach," IEEE Trans. Geosci. Remote Sens., vol. 50, no 5, pp. 1986-2000, May 2012.

[10] F. Liang, F. Qi, Q. An, H. Lv, F. Chen, Z. Li, and J. Wang, "Detection of multiple stationary humans using UWB MIMO radar," Sensors, vol. 16, pp. 1922-1938, Nov. 2016.

[11] Y.S. Yoon, and M. G. Amin, "High-resolution through-the-wall radar imaging using beamspace MUSIC," IEEE Trans. Antennas Propag., vol. 56, no 6, pp. 1763-1774, Jun. 2008.

[12] X. P. Masbernat, M. G. Amin, F. Ahmad, and C. Ioana, "An MIMO-MTI approach for through-the-wall radar imaging applications," in Proc. 5th Int. Waveform Diversity and Design Conf., Niagara Falls, ON, Canada, 2010, pp. 188-192.

[13] F. Ahmad, and M. G. Amin, "Wall clutter mitigation for MIMO radar configurations in urban sensing," in Proc. 11th Int. Conf. on Information Science, Signal Processing and their Applications, Montreal, QC, Canada, 2012, pp. 1165-1170.

[14] M. G. Amin, and F. Ahmad, "Compressive sensing for through-the-wall radar imaging," J. Electron. Imaging, vol. 22, no 3, pp. 1-21, Jul.-Sep. 2013.

[15] Y. Yu, F. Ahmad, A. P. Petropulo, and M. G. Amin, "CS-MIMO radars for through-the-wall imaging in an indoor multipath environment," in Proc. SPIE vol. 9109, Compressive Sensing III, May 2014, pp. 61-69.

[16] S. Pisa, E. Piuzzi, E. Pittella, P. D’Atanasio, A. Zambotti, and G. Sacco, "Comparison between delay and sum and range migration algorithms for image reconstruction in through-the-wall radar imaging systems," IEEE 
J. of Electromagn., RF, Microw. Med. Biol., vol. 2, no 4, pp. 270-276, Dec. 2018.

[17] A. Quinquis, E. Radoi, and F.-C. Totir, "Some radar imagery results using superresolution techniques," IEEE Trans. Antennas Propag., vol. 52, no 5, pp. 1230-1244, May 2004

[18] P. D’Atanasio, A. Zambotti, S. pisa, E. Pittella, and E. Piuzzi, “Complex permittivity measurements for moisture and salinity characterization of building materials," in Proc. Int. Conf. on Metrology for Archeology and Cultural Heritage, Lecce, Italy, Oct., 2017, pp. 437-451.

[19] S. Stavrou and S. R. Saunders, "Review of constitutive parameters of building materials," in Proc. 12 $2^{\text {th }}$ Int. Conf. on Antennas and Propagation, Exeter, U.K., 2003, pp. 211-215.

[20] T. Dogaru, L. Nguyen, and C. Le, "Computer models of the human body signature for sensing through the wall radar applications," US Army Research Laboratory, Adelphi, MD, Sep. 2007.

[21] E. Piuzzi, P. D’Atanasio, S. Pisa, E. Pittella, and A. Zambotti, "Complex radar cross section measurements of the human body for breath activity monitoring applications," IEEE Trans. Instrum. Meas., vol. 64, no 8, pp. 2247- 2258, Aug. 2015.

[22] C. A. Balanis, "Advanced Engineering Electromagnetics," Wiley, Hoboken, NJ, USA, 2012.

[23] F. H. C. Tivive, A. Bouzerdoum, and M. G. Amin, "A subspace projection approach for wall clutter mitigation in through-the-wall radar imaging," IEEE Trans. Geosci. Remote Sens., vol. 53, no 4, pp. 21082122, Apr. 2015.

[24] Y. S Yoon, and M. G. Amin, "Spatial filtering for wall-clutter mitigation in through-the-wall radar imaging," IEEE Trans. Geosci. Remote Sens., vol. 47, no 9, pp. 3192-3208, Sep. 2009.

[25] M. Dehmollaian and K. Sarabandi, "Refocusing through building walls using synthetic aperture radar," IEEE Trans. Geosci. Remote Sens., vol. 46, no 6, pp. 1589-1599, Jun. 2008.

[26] R. Agliata; L. Mollo; and R. Greco, "Use of TDR to compare rising damp in three tuff walls made with different mortars," J. Mater. Civ. Eng., pp. 1-7, Nov. 2016.

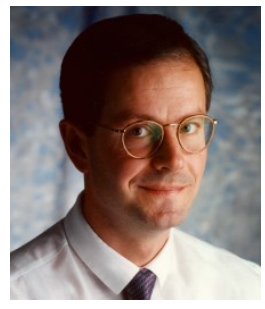

Renato Cicchetti (S'83-M'83-SM'01) was born in Rieti, Italy, in May 1957. He received the Laurea degree in electronics engineering (summa cum laudee) from the University of Rome "La Sapienza," Rome, Italy, in 1983. From 1983 to 1986, he was an Antenna Designer at Selenia Spazio S.p.A. (now Thales Alenia Space S.p.A.), Rome, Italy, where he was involved in studies on theoretical and practical aspects of antennas for space application and scattering problems. From 1986 to 1994, he was a Researcher, and from 1994 to 1998, he was an Assistant Professor at the Department of Electronics Engineering, University of Rome "La Sapienza," where he is currently a Full Professor. In 1998, 2002, and in 2006 he was Visiting Professor at the Motorola Florida Corporate Electromagnetics Research Laboratory, Fort Lauderdale, FL, where he was involved with antennas for cellular and wireless communications. In 2012-2016 he was the Lead Editor of the Special Issue on "Wideband, Multiband, Tunable, and Smart Antenna Systems for Mobile UWB Wireless Applications" for the International Journal of Antennas and Propagation. His current research interests include electromagnetic field theory, asymptotic techniques, electromagnetic compatibility, wireless communications, microwave and millimeter-wave integrated circuits, and antennas. Dr. Renato Cicchetti is a Senior Member of the Institute of Electrical and Electronic Engineers, of the Italian Electromagnetic Society (SIEm) and he results listed in Marquis Who's Who in the World and Who's Who in Science and Engineering.

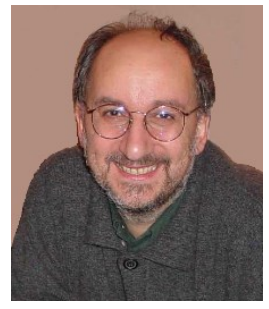

Stefano Pisa (M'91-SM'16) received the Electronic Engineering and Ph.D. degrees from the University of Rome "La Sapienza," Rome, Italy, in 1985 and 1988, respectively. In 1989, he joined the Department of Information Engineering, Electronics and Telecommunications (DIET), Sapienza University of Rome as a Researcher. Since 2001, he has been an Associate Professor with the same university. His research interests are the interaction between electromagnetic fields and biological systems, therapeutic and diagnostic applications of electromagnetic fields, medical applications of radar, and the modeling and design of microwave circuits. He has authored over 180 scientific papers. He serves as a reviewer for various international journals. Prof. Pisa is a Senior member of the IEEE
Microwave Theory and Techniques Society, a Senior Member of the International Union of Radio Science (URSI) and a member of the Italian Society of Electromagnetics (SIEm).

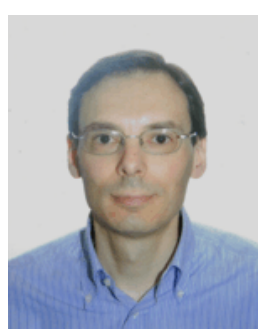

Emanuele Piuzzi (M'09) received the M.S. (cum laude) and Ph.D. degrees in electronic engineering from the Sapienza University of Rome, Rome, Italy, in 1997 and 2001, respectively. He is currently an Associate Professor with the Department of Information Engineering, Electronics and Telecommunications, Sapienza University of Rome, where he is engaged in teaching electrical measurements. $\mathrm{He}$ is the coauthor of over 140 publications. His current research activities include the measurement of dielectric characteristics of fluids and granular materials through time- and frequency-domain reflectometry approaches, impedance pneumography, ultrawideband radar techniques for the monitoring of cardiopulmonary activity in patients, and electrical impedance tomography. Dr. Piuzzi is a member of the IEEE Instrumentation and Measurement Society, of the Italian Group of Electrical and Electronic Measurements (GMEE), and of the Italian Electrotechnical Committee (CEI). He serves as a reviewer for different international journals.

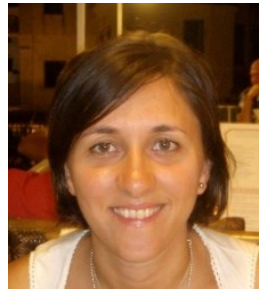

Erika Pittella (M'17) received the M.S. (cum laude) and Ph.D. degrees in electronic engineering from the Sapienza University of Rome, Rome, Italy, in 2006 and 2011, respectively. She is currently Assistant Professor at Pegaso University (Naples, Italy). Her research interests are related to the measurement of complex permittivity of materials, time domain reflectometry applications and biomedical instrumentation design. Her research interests also include the modeling of ultrawideband radars for the remote monitoring of cardiorespiratory activity and the design of sources, antennas, and receivers of such systems. Dr. Pittella has co-authored over 60 articles and she is an Associate Editor of IET Microwaves, Antennas, and Propagation. She is a member of the IEEE Instrumentation and Measurement Society and of the Italian Group of Electrical and Electronic Measurements (GMEE).

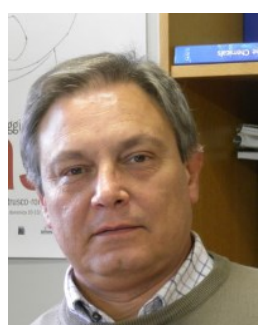

Paolo D'Atanasio was born in Rome, Italy, in 1959. He received the Full Degree (summa cum laude) in Physics from the Sapienza University of Rome, Rome, Italy, in 1986. He joined the Italian National Agency for New Technologies, Energy and Sustainable Economic Development, Rome, as a Researcher, in 1988. Since 2000, he is responsible of the Electromagnetic Compatibility Laboratory and since 2011 he is Director of Research. His current research interests include electromagnetic measurements of radar cross section, antenna characterization, dielectric spectroscopy measurements, EMC/EMI tests for electric vehicles, interaction between electromagnetic fields, and biological systems. Dr. D'Atanasio is a member of the Italian Society of Physics and the Italian Electrotechnical Committee.

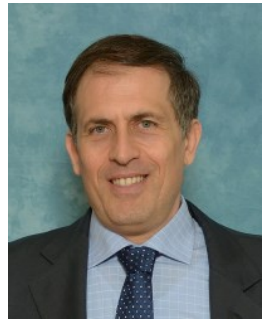

Orlandino Testa received the Laurea degree (cum laude) in electronic engineering degree and the Ph.D. degree from the University of Rome "La Sapienza", Rome, Italy, in 1997 and 2003, respectively. Since 2001, he has been a high school Teacher at the I.T.I.S. "G. Armellini" Institute of Rome, where he is involved in teaching electronics and telecommunications. $\mathrm{He}$ is also currently collaborating with the Department of Electronic Engineering, University of Rome "La Sapienza." At present, he is studying high-frequency models for the analysis of radio coverage in indoor environments and tunnels with particular attention to EMC/EMI problems. His main research interests are propagation and radiation of electromagnetic fields, electromagnetic compatibility, microwave and millimeter-wave integrated circuits, and antennas. 